\title{
Studies on Structural, Morphological, Local Electrical, Optical and Magnetic Properties of Iron Site Manganese Substituted Yttrium Orthoferrite
}

Turghunjan Gholam ( $\nabla$ turghunjan@stu.xmu.edu.cn )

Xiamen University College of Physical Science and Technology

\section{Liu Peng}

Institute of High Energy Physics Chinese Academy of Sciences

Dong Yu-Hui

Institute of High Energy Physics Chinese Academy of Sciences

Wang Hui-Qiong

Xiamen University College of Physical Science and Technology

\section{Research Article}

Keywords: Yttrium orthoferrite, Fe-site, Hydrothermal method, X-ray absorption fine structure

Posted Date: December 1st, 2020

DOl: https://doi.org/10.21203/rs.3.rs-113350/v1

License: (a) (i) This work is licensed under a Creative Commons Attribution 4.0 International License.

Read Full License 


\section{Abstract}

Yttrium orthoferrite $\left(\mathrm{YFeO}_{3}\right)$ is of considerable interest for its potential application in magnetic field sensors and magneto optical data storage devices. Doping is one of the effective approaches to tune the compound properties. And it is important to determine the doping sites of the dopants to better understanding the related mechanism. In this work, Manganese $(\mathrm{Mn})$ doped $\mathrm{YFeO}_{3}$, i.e., $\mathrm{YFM}_{x} \mathrm{O}$ powders with $0 \leq x \leq 0.1$ were synthesized by hydrothermal method to study the influences of doping on their structural, morphological, local electrical, optical and magnetic properties. The experimental results show that $\mathrm{Mn}$ dopants occupy Iron $(\mathrm{Fe})$ sites and that all these samples exhibit an orthorhombic structure with space group Pnma. Refined structure parameters are presented. Morphology images show the shape evolution from layered to multilayered with increasing $\mathrm{Mn}$ content. The Fe and $\mathrm{Y} K$ K-edge local structure studies indicate that the valency of $F e$ and $Y$ is mainly found in trivalent state, which also indicate that substitution of $\mathrm{Mn}$ ions not only affects the nearest neighbor atomic shell of Fe but also affects the nearest neighbor's local structure of $Y$ atoms. IR spectra reveal the characteristic vibrations of the obtained $\mathrm{YFM}_{x} \mathrm{O}$ samples. From the magnetic study, it is observed that the substitution of $\mathrm{Y}$ ions by $\mathrm{Mn}$ ions changes the magnetic property of $\mathrm{YFeO}_{3}$ from ferromagnetic to paramagnetic. Our results show that the addition of Mn exhibits an evident influent on the local structural and magnetic properties.

\section{Introduction}

The second-generation multiferroic materials, i.e., rare earth orthoferrites $\left(\mathrm{RFeO}_{3}, \mathrm{R}=\right.$ rare-earth ions or $\left.\mathrm{Y}\right)$, have been extensively studied for decades owing to their multiferroic, spin-switching, and magnetooptical properties [1-3]. Some other outstanding features of these materials, such as high domain wall velocity and the existence of Bloch lines [4], have promising applications in sensors, information storage, and spintronics, etc $[5,6]$. In most cases, $\mathrm{RFeO}_{3}$ is crystallized by the corner-linked $\mathrm{FeO}_{6}$ octahedral forming a three-dimensional network in a centrosymmetric Pbnm (or Pnma) unit cell [7]. That is to say, the unit cell consists of four molecules with the $\mathrm{R}^{3+}$ cations located on the center and the $\mathrm{Fe}^{3+}$ ions are nearly octahedrally coordinated to six $\mathrm{O}^{2-}$ ions [8]. Unlike the first generation multiferroics, $\mathrm{RFeO}_{3}$ not only combines antiferromagnetic and ferroelectric orders but also show magnetoelectric coupling effects [9$11]$.

With the rise of $\mathrm{RFeO}_{3}$ materials in recent years, as a member of them, yttrium orthoferrite $\left(\mathrm{YFeO}_{3}\right.$, short for YFO) has attracted much attention from the research community because of its magnetic, physical, and chemical properties due to the ionic and electronic defects as well as structure distortions [12]. YFO crystallizes in the perovskite structure with the $\operatorname{Pnma}\left(\mathrm{D}_{2 \mathrm{~h}}{ }^{16}\right)$ space group [13]. Despite the centrosymmetric nature, this material with its low Curie temperature $T_{\mathrm{C}} \sim 256^{\circ} \mathrm{C}$ and high Neel temperature $T_{\mathrm{N}} \sim 370^{\circ} \mathrm{C}$, can exhibit both ferroelectric and antiferromagnetic behaviors [14, 15]. Among all the $\mathrm{RFeO}_{3}$ materials, YFO has been most thoroughly investigated. Doping with different types of ions is a very powerful way to modify, and enhance the properties of the compound. The substitution of 
manganese $(\mathrm{Mn})$ for iron $(\mathrm{Fe})$ is most interesting because the electron configuration of $\mathrm{Mn}^{3+}$ and $\mathrm{Fe}^{3+}$ ions are quite different and effective magnetic moments are different for both these ions. Kwanghee et al. [16] studied the absence of ferroelectricity and the origin of depolarization currents in $\mathrm{YFe}_{0.8} \mathrm{Mn}_{0.2} \mathrm{O}_{3}$. Crystal growth, spin reorientation, magnetic anisotropy, and thermal properties of $\mathrm{YFe}_{0.8} \mathrm{Mn}_{0.2} \mathrm{O}_{3}$ and $\mathrm{YFe}_{0.6} \mathrm{Mn}_{0.4} \mathrm{O}_{3}$ single crystals have been studied by Xie et al. $[17,18]$. Dielectric relaxation, electric modulus, ac conductivity, structural and magnetic properties of Mn-doped YFO have been investigated by Zhang et al. [19, 20]. Bipul et al. [21, 22] reported the magnetic, dielectric properties, and exchange bias in Mn-doped YFO. Cao et al. [23] attempted to improve the conductivity of YFO by substituting Mn for Fe. Attila et al. [24] highlighted some structural, electric, and magnetic properties of $\mathrm{YMn}_{1}-{ }_{x} \mathrm{Fe}_{x} \mathrm{O}_{3}$. The influence of $\mathrm{Mn}$ on the structure and magnetic properties of YFO nanocrystal has been studied by Shen et al. [25]. Mandal et al. [26] reported the observation of magnetoelectric and magnetodielectric effects at different temperatures in $\mathrm{YFe}_{1-x} \mathrm{Mn}_{x} \mathrm{O}_{3}$. ${ }^{57} \mathrm{Fe}$ Mossbauer spectrometry and magnetic susceptibility of polycrystalline $\mathrm{YMn}_{1-} \mathrm{Fe}_{x} \mathrm{O}_{3}$ compounds have been measured by Zaghrioui et al. [27].

However, the local electronic structure of Mn-doped YFO has rarely been investigated. To address this issue, we have studied the local structure properties of Fe-site Mn-doped YFO powders, using an X-ray absorption fine structure (XAFS) spectroscopy technique. The result of absorption spectra comes from the removal of a core electron when an X-ray photon is absorbed. The emitted photoelectrons are backscattered by the neighboring atoms. As it is well known, XAFS has proven to be one of the best tools to probe the local electronic environment each absorbing atom in simple or complex systems. As a structural tool, extended X-ray absorption fine structure (EXAFS) is capable of determining atomic arrangements, which could provide a reliable structural parameters information. EXAFS can directly measure the radial displacement between an absorbing atom and its neighbors [28]. However, in some cases, the accuracy of the structural determinations obtained from EXAFS can be inconclusive, such as the well-known limitations of the fitting methodology to determine coordination numbers and polyhedral environments [29]. Fortunately, X-ray absorption near edge structure (XANES) can fill these holes, which is very sensitive to the geometrical details of the absorbing atoms, e.g., formal oxidation state, specific symmetry, coordination chemistry, and bond angles, etc [30]. In our work, both EXAFS and XANES were examined at the $K$-edges of $Y$ and Fe. We have also studied the morphological, optical, and magnetic properties of the target compound.

\section{Materials And Methods}

\subsection{Sample preparation}

$\mathrm{YFe}_{1-x} \mathrm{Mn}_{x} \mathrm{O}_{3}\left(\mathrm{YFM}_{x} \mathrm{O}\right)$ powders with $0 \leq x \leq 0.1$ were prepared by a hydrothermal synthesis process. High purity of yttrium nitrate $\left(\mathrm{Y}\left(\mathrm{NO}_{3}\right)_{3} \cdot 6 \mathrm{H}_{2} \mathrm{O}, 99.9 \%\right)$, iron (III) nitrate $\left(\mathrm{Fe}\left(\mathrm{NO}_{3}\right)_{3} .9 \mathrm{H}_{2} \mathrm{O}, 99.9 \%\right)$, manganese (II) chloride $\left(\mathrm{MnCl}_{2} \cdot 4 \mathrm{H}_{2} \mathrm{O}, 99.9 \%\right)$ and potassium hydrate $(\mathrm{KOH}, 95 \%)$ were used as the starting materials. In the typical procedure, the $\mathrm{Y}\left(\mathrm{NO}_{3}\right)_{3} \cdot 6 \mathrm{H}_{2} \mathrm{O}, \mathrm{Fe}\left(\mathrm{NO}_{3}\right)_{3} \cdot 9 \mathrm{H}_{2} \mathrm{O}$, and $\mathrm{MnCl}_{2} \cdot 4 \mathrm{H}_{2} \mathrm{O}$ were dissolved into $30 \mathrm{ml}$ deionized water under magnetic stirring for $10 \mathrm{~min}$ until the clear solution was obtained. Then $0.75 \mathrm{~mol}$ 
of solid $\mathrm{KOH}$ as the mineralizing agent was directly added into the mixture solution under magnetic stirring for $30 \mathrm{~min}$. Those solutions were subsequently transferred into the Teflon-lined autoclave and heated for $72 \mathrm{~h}$ at $240{ }^{\circ} \mathrm{C}$. After the hydrothermal reaction was complete, the resultant compounds were cooled to room temperature naturally. Finally, the products were washed several times with both ethanol and distilled water and then air-dried for $8 \mathrm{~h}$ at $80^{\circ} \mathrm{C}$.

\subsection{Characterization}

Phase purity and crystal structure were characterized by X-ray diffraction (XRD) on the Mac Science M18XHF22-SRA X-ray diffractometer using Cu Ka radiation $(\lambda=1.5406 \AA)$. Rietveld refinement of the samples was performed using the GSAS-EXPGUI program. The scanning electron microscope (SEM) and energy dispersive spectroscopy (EDS) images were used to determine the shape, morphology, and composition of the samples using LE01430VP equipment. XAFS spectra were recorded at the Beamline 1W2B of the Beijing Synchrotron Radiation Facility (BSRF), the Institute of High Energy Physics, China. Pellets prepared with boron nitride to optimize the thickness were used and put on tape for the detection in the whole experiment. XAFS spectra at the Fe and $Y K$-edges were collected in the transmission mode. Data elaboration has been performed using the ATHENA code of the IFEFFIT software package for analysis [31]. $E_{\mathrm{o}}$ was defined as the maximum of the first derivative of the absorption edge. The atomic absorption data were a transition from energy $E$ space to wave vector $K$ space. A Fourier transform was performed to obtain a distribution function around the absorbing atom in radial distance $R$ space. An isolated single shell $\chi(k)$ was obtained by back transformation of the first shell signal from $R$ space to $K$ space. The $\chi(k) k^{3}$ of the EXAFS spectra were calculated in the interval $K=0.0$ to $14.0 \AA^{-1}$ and the Fourier transform of $\chi(k) k^{3}$ signals were taken in the $R=0.00$ to $10.0 \AA$, using with a Hanning window function. The absorption profiles of iron oxide $\left(\mathrm{Fe}_{2} \mathrm{O}_{3}\right)$ and yttrium oxide $\left(\mathrm{Y}_{2} \mathrm{O}_{3}\right)$ were measured as references for valence state comparison. IR measurements were performed with Fourier transform infrared spectra (FTIR) using the EQUINOX55 spectrometer. A vibrating sample magnetometer (VSM, MPMS-XL-7) was applied to measure the magnetic properties.

\section{Results And Discussion}

\subsection{Crystal structure and Rietveld refinement}

Figure 1 presents the typical XRD patterns of pure YFO and $\mathrm{YFM}_{x} \mathrm{O}(0 \leq x \leq 0.1)$ powders at room temperature. The XRD patterns indicate a pure phase for all samples. Our study shows that YFO can be synthesized via hydrothermal method without having common impurity phases like $\mathrm{Y}_{2} \mathrm{O}_{2}$ and $\mathrm{Fe}_{2} \mathrm{O}_{3}$. As it is expected, no characteristic peaks related to $\mathrm{Mn}$ in the XRD patterns in comparison with the standard XRD patterns of pure YFO (orthorhombic Pnma: JCPDS file No. 01-086-0171), which is reasonable to suppose that all Mn ions have entered into the Fe-site of YFO. As is shown in the inset Fig. 1(a) that there is a slight shift of the major peak (200) to a bigger 2theta angle with the increase of Mn concentration. From the enlarged spectra in Fig. 1(b), other main peaks, such as (311) and (321) also have the same shifting trend. This shift in the diffraction angle might be ascribed to the unit cell contraction or the 
decrease in lattice constants because the ion radius of $\mathrm{Mn}^{3+}\left(r_{\mathrm{Mn}}=0.580 \AA\right)$ is smaller than that of $\mathrm{Fe}^{3+}$ ion $\left(r_{\mathrm{Fe}}=0.645 \AA\right)$. However, it is worth noting that the intensity of the diffraction peaks reduced and merged partially to form broadened peaks after the Mn concentration further increased, especially for the sample with $x=0.1$ (see in Fig. 1(a) and (b)), which is attributed to the presence of distortions, and a diminution in the crystalline size [32]. From the crystallography point of view, the intensity of the peaks is usually related to the crystallinity, thus, the broadened width of the XRD peaks with the increase of Mn content indicates the decreasing crystallinity of $\mathrm{YFM}_{x} \mathrm{O}$. The reduced crystallinity may be due to the reason that $\mathrm{Mn}^{3+}$ favors the creation of more nucleation sites, which in turn inhibits the growth of crystal grains.

To quantify the structure in detail and determine lattice parameters of the samples, an analysis of the XRD patterns by the Rietveld refinement was done using the Pnma space group in the orthorhombic unit cell. However, it is confirmed from the XRD results that the diffraction profiles belong to the orthoferrite structure for all samples. For such reason, only the samples with $x=0$ and $x=0.1$ were analyzed by Rietveld refinement, as shown in Fig. 2(a)-(b). Figure 2(c) shows the common crystal structure of the YFO. In this structure, $\mathrm{Y}^{3+}$ is surrounded by $12 \mathrm{O}^{2-}$ ions, and $\mathrm{Fe}^{3+}$ is surrounded by six $\mathrm{O}^{2-}$ ions arranged in $\mathrm{FeO}_{6}$ octahedra. Table 1 in Fig. 2 shows the variation of unit cell parameters $v s$ the content of $\mathrm{Mn}$ in $\mathrm{YFM}_{x} \mathrm{O}$ with $x=0$ and $x=0.1$. According to refined values, lattice parameters of YFO are $a=5.5944 \AA, b=$ $7.6123 \AA$, and $c=5.2812 \AA$ and they are comparable to those reported in works of literature [33, 34]. The goodness of fit $\chi^{2}$ is the conformity between an experimental result and theoretical expectation. $R$ values are useful indicators for the evaluation of a refinement. As it is known, the Rietveld refinement results are reliable when the $R_{w p}$ value is less than $10 \%$ and the $\chi^{2}$ in the range of $1.0 \sim 1.3$. Small values of $R$ factors indicate good consistency between observed and calculated results in XRD patterns, which means high reliability in our Rietveld refinement of $\mathrm{YFM}_{\chi} \mathrm{O}$ powders. As can be observed, the values of the lattice parameters, $a, b$ and $c$, are decreased with increasing Mn content, leading to the contraction of the unit cell volume, because of the smaller ionic radius of $\mathrm{Mn}$ with respect to $\mathrm{Fe}$, a similar phenomenon has been observed in $\mathrm{Y}$ doped $\mathrm{BiFeO}_{3}[35]$. The variations in the intensity of peaks and lattice parameters can be attributed to the incorporation of the dopant in the crystal [36]. Thus, it is reasonable to believe that $\mathrm{Mn}^{3+}$ ions are introduced to the iron sites of YFO, which can also be further illustrated by EDX results.

\subsection{Morphological evolution and composition}

The morphology and phase structure of the pure YFO and $\mathrm{YFM}_{x} \mathrm{O}$ powders are investigated by SEM micrographs, as shown in Fig. 3(a)-(e). From these images, we can observe that the size average (ca. $10 \mu \mathrm{m})$ is nearly the same in all the particles with different shapes, except for the sample with $x=0.1$. It is observed that the pure YFO exhibit a layered cuboid shape. When the Mn content $x=0.025$, the multilayered cuboid is observed, and it is continuously layered with further doping (see Fig. 3(b)-(d)). In the hydrothermal crystallization processes of $\mathrm{RFeO}_{3}$, the addition of $\mathrm{KOH}$ could transfer $\mathrm{R}$ and $\mathrm{Fe}$ ions into amorphous hydroxides $\mathrm{R}(\mathrm{OH})_{3}$ and $\mathrm{Fe}(\mathrm{OH})_{3}$ for a very short time [37]. The formation of $\mathrm{RFeO}_{3}$ can be 
described by the chemical reactions, as follows: $\mathrm{R}^{3+}+\mathrm{OH}^{-}=\mathrm{R}(\mathrm{OH})_{3}(\mathrm{~s}) ; \mathrm{Fe}^{3+}+\mathrm{OH}^{-}=\mathrm{Fe}(\mathrm{OH})_{3}(\mathrm{~s})$. The transition metal or rare-earth hydroxides usually form layered structures with ions inserted between the layers of metal hydroxide [38], which is in good agreement with the results observed by SEM. In contrast, when the Mn content reaches $x=0.1$, the grain morphology changes to larger agglomerates shape with a remarkably reduced grain size. The larger agglomerates are composed of smaller particles. From the ionic radii point of view, smaller Mn ions can enter the Fe-site of YFO, which maintains the charge balance in the system. After Mn doping, mass transportation becomes weaker and the grain growth is inhibited. A similar reduction of particle size can be found in our previous work [39]. Figure 3(f) presents schematic illustrations of the morphological evolution of $\mathrm{YFM}_{x} \mathrm{O}$ powders. This result indicates that the crystal shapes of $\mathrm{YFM}_{x} \mathrm{O}$ powders are strongly dependent on the concentration of $\mathrm{Mn}$. The nucleation rate of the grains has changed when the Mn concentration exceeds a certain value, and this has resulted in a different grain morphology, from which it can be concluded that YFO is a suitable compound to study the shape-dependent physical properties. Elemental compositions, as determined by EDS analysis, are shown in Fig. 3(g)-(k). The obtained pure YFO (see Fig. 3(a)) reveals the existence of elemental Y, Fe, and O. The corresponding EDS patterns of $\mathrm{YFM}_{X} \mathrm{O}$ (see Fig. 3(h)-(k)) shows the characteristic peaks belong to the $\mathrm{Y}$, $\mathrm{Fe}, \mathrm{Mn}$, and $\mathrm{O}$, indicating the well doped of $\mathrm{Mn}$ in YFO. The spectra reveal that the higher Mn-doped molar ratio leads to the larger existence of Mn in YFO powders. To have a better comprehension of the structural evolution of $\mathrm{YFM}_{X} \mathrm{O}$, we have performed XAFS analysis on all samples.

\subsection{Fe and $Y K$-edge local electronic structure}

Figure 4 shows the $\mathrm{Fe} K$-edge XANES spectra of the pure $\mathrm{YFO}$ and $\mathrm{YFM}_{x} \mathrm{O}$ with $\mathrm{Fe}_{2} \mathrm{O}_{3}$ as a standard compound. As seen in Table 2 in Fig. 4, for all powder samples, the absorption edge energies were found at $7127 \mathrm{eV}$ (less than $0.5 \mathrm{eV}$ error) which is close to the absorption edge energy of reference $\mathrm{Fe}_{2} \mathrm{O}_{3}$ at $c a$. $7127.53 \mathrm{eV}$. The valence state of $\mathrm{Fe}_{2} \mathrm{O}_{3}$ is $\mathrm{Fe}^{3+}$, which means the $\mathrm{Fe}$ atoms in all $\mathrm{YFM}_{x} \mathrm{O}$ samples have the oxidation states of $3+$ and all doping samples are dominated by the original orthorhombic Pnma phase without charge transfer from the Fe cations. Besides, the pre-edge peak position shifts towards higher energy with increasing oxidation state [40]. In our samples, there is no pre-edge peak position shift, which further proves Fe is in $3+$ valance state in all the samples. The XANES spectra of all these samples show an analogous pattern to each other, which substantiates the fact that $\mathrm{Mn}$ ions have occupied $\mathrm{Fe}$ sites of YFO. The invisible pre-edge peaks are observed in the spectra of both pure YFO and YFM $O$ samples. The pre-edge peak is usually related to quadrupole transition from $1 s$ core state to $3 d$ empty state, which is expected to be very weak for a Fe cation in an octahedral environment [41]. It is well known that the pre-edge peak is a fingerprint of the octahedral coordination of Fe. Our spectra show almost no pre-edge shift as a function of $x$ but their intensity is changing with $x$. In the enlarged XANES spectra in Fig. 4(a), it can be seen that, in the beginning, the intensity of the pre-edge peak is slightly increased. For $0.025<x<0.1$, the intensity of the pre-edge peak begins to decrease and it is largest for the $x=0.1$ sample. It is worth noting that for the compounds with $x=0.1$ the pre-peak increases its intensity with $x$, which possibly indicates a decrease of the symmetry of the Fe environment. A similar phenomenon has 
been observed in the other perovskite $\mathrm{ABO}_{3}$ system $[42,43]$. The increasing intensity in the pre-edge peak indicates the enhancement of the $1 s-3 d$ electric dipole-forbidden transition while decreasing the intensity caused by the $1 s-4 p$ dipole-allowed transition. These transitions have caused by $\mathrm{Mn}$ substitution, indicating a distortion of $\mathrm{FeO}_{6}$ octahedron. The two post-edge peaks are attributed to the transfer of $2 p$ electrons in the oxygen $2 p$ band to the Fe $3 d$ orbital by a shakedown process [44]. The intensity of these two post-edge peaks first increases then decreases when $x=0.1$, as shown in Fig. 4(b). This indicates that the $3 d-4 p$ transition and charge transfer from the $02 p$-Fe $3 d$ is enhanced with both low and high doping contents of Mn due to the loss of inversion octahedral symmetry of the oxygens around the Fe atoms [45]. These evolutions indicate that the local geometry and structure of Fe have changed.

In addition to the XANES data above, further analysis is carried out using the EXAFS. The Fourier transformation of the EXAFS is also shown. EXAFS features could provide useful information on both the short-range and the long-range orders (i.e., in the first shell and higher shell than the second). Figure 5 shows the variation of the observed $k^{3}$-weighted EXAFS oscillation of the YFM ${ }_{x} O$ powders. The error noise is observed above ca. $9 \AA^{-1}$. Oscillations are still visible above ca. $12 \AA^{-1}$, being less intense at the higher $k$, and show clear evolution as a function of Mn concentration, as given in Fig. 5(a). This phenomenon may be related to the less symmetric environment around Fe cations. The changes in the local structure could be better revealed in the Fourier transforms of the EXAFS oscillations providing real space information. The Fourier Transforms of the $k^{3}$-weighted EXAFS spectra of the YFM ${ }_{x} 0$ samples are shown in Fig. 6 and the detailed coordination distances are listed in Table 3 in Fig. 6 . The first and the second neighbor distributions in distance are easier to separate from the other shells in the Fourier transform. There are some characteristics peaks in spectra: (1) There are two strong amplitude peaks between $1 \AA$ and $4 \AA$, with the first peak located at $1.54 \AA$, which is corresponding to the Fe- 0 coordinate peak due to the first oxygen coordination sphere of Fe ions. (2) The second strong peak is located at $3.28 \AA$, which is known as the Fe-Fe/Mn peak caused by the second rate nearby metal ions. (3) The small intensity of other peaks is not yet clear. They are probably due to the multiple scattering processes in the first coordination shell. Compared to the pure YFO, there is almost no shift of peak position (see Table 3 in Fig. 6) but the intensity of the Fe-O peak is decreased with the Mn content increases, as shown in Fig. 6(a). Moreover, with a close look at the Fe-O peak, we can observe that the intensity of this peak first decreases $(x=0.025)$ then slightly increases $(x=0.05)$ and then decreases again when $x$ beyond 0.05 . The reduction of the Fe-O peak intensity represents the loss of short-range order in the system. The intensity of the Fe-Fe/Mn peak also has the same trend (see Fig. 6(b)), which is due to the change of the local structure from Fe-Fe into Fe-Fe/Mn.

The normalized $Y K$-edge XANES spectra of the studied samples $Y F M{ }_{x} \mathrm{O}$, including the reference $\mathrm{Y}_{2} \mathrm{O}_{3}$ compound, are shown in Fig. 7. All the samples showed nearly similar near-edge features, indicating a similar local structure around $Y$ ion in the first shell. The $Y K$-edge XANES spectra of all samples are similar to those of Fe K-edge XANES spectra without pre-edge peaks. Generally, the shift of the absorption edge energy in XANES is sensitive to the oxidation state of $Y$ in the material. The edge position of the $\mathrm{Y}_{2} \mathrm{O}_{3}$ standard is approximately $17044.65 \mathrm{eV}$, while those of $\mathrm{YFM}_{x} \mathrm{O}$ samples are approximately $17044 \mathrm{eV}$ 
(less than $0.5 \mathrm{eV}$ error). These can be used simply as a fingerprint of phases and valence states, from which it can be seen that the edge positions of Mn-doped samples are quite similar to that of the standard sample of $\mathrm{Y}_{2} \mathrm{O}_{3}$. Thus, this result indicates that the $\mathrm{Y}$ ions in our samples are in the $3+$ valence state. All samples show no pre-edge peaks, which is associated with the $1 s$ to $4 d$ transition of $\mathrm{Y}$. More specifically, this transition is partially allowed for the distortion of octahedral, only when $p$ orbitals are mixed with $d$ orbitals. The fact that this transition is not observed indicates a small distortion of the octahedral symmetry. From the examination of Table 4 in Fig. 7, it is clear that with Mn concentration increasing, there is no evident shift of the absorption edges in the whole series, but their intensity shows some difference. The two main peaks can be observed for all samples, which could be due to the transition from $1 s$ state to $5 p$ state [46]. For better clarity, the enlarged post-edge peak is shown in Fig. 7(a), from which it can be seen that the intensity of the first strong post-edge peak decreases as Mn content increases. As for the second strong post-edge peak, highest intensity can be found for $x=0.025$ and $x=0.075$ samples and lowest for $x=0.1$ sample. The evolution of the post-edge peak intensity indicates that the local structure of $\mathrm{Y}$ has changed. Although $\mathrm{Mn}$ is doped in the Fe-site, the $\mathrm{Y}$-site atom is also affected by the substitution.

Along the main edge, the XAFS spectra show a typical oscillation, which is caused by the scattering process of the electron wave near the nearest neighboring atoms. After the standard data processing, these waves yield of the $\chi(k) k^{3}$ function and its Fourier transformation data could provide valuable information about the coordination number, nearest neighbor distances, and the coordination geometry, etc. Among them, the radial distribution function is generated by backscattering along the $R$ axis of the atom at different distances. This indicates the distance between the atom level and the central absorption atom. The analyzable $k$ range of the EXFAS data is from 0 to $14 \AA^{-1}$ and the spectra are weighted by $k$ to amplify the oscillations at high $k$. The $Y K$-edge $k^{3}$-weighted EXAFS curves of the YFM $\mathrm{F}_{x} \mathrm{O}$ samples are given in Fig. 8. All the oscillations show similar patterns in the higher and lower $k$ (see Fig. 8(a)), which indicates that the EXAFS functions of the $Y$ atoms seem unchanged in all samples. The Fourier transforms of the $k^{3}$-weighted EXAFS functions of the powder samples YFM ${ }_{x} O$ are shown in Fig. 9. From the figure, it can be seen that the first and the second neighbor distributions are well separated from each other and other shells in the whole spectra. The primary features are two dominant peaks and other small peaks for all samples distributed at different distances: (1) The first shell has an $R$ of $1.17 \AA$, corresponding to the $Y-0$ peak caused by scattering of oxygen anions from the nearest neighboring $Y$ atomic shell. (2) The second shell with an $R$ of $2.54 \AA$, corresponding to the $Y$-Y peak, which can be explained by the scattering of oxygen anions from the next nearest neighboring $Y$ atomic shell. The low second peak is a common feature in the case of EXAFS, which is often explained as the presence of high levels of disorder in the materials. (3) The other small peaks are probably due to a large number of multiple scattering in the first shell. More details are shown in Table 5 in Fig. 9, where the peak positions of $\mathrm{YFM}_{X} \mathrm{O}$ samples remain almost unchanged but the intensities of which are affected by $\mathrm{Mn}$ substitution. Compared to pure YFO, the intensity of the $\mathrm{Y}-\mathrm{O}$ peak is increased then largely decreases for $x=0.1$ sample. More specifically, the intensity of Y-O peak first increases with $x=0.025$ then a little decrease when $x=0.05$ and then increases again for $x=0.075$, as shown in Fig. 9(a). Unlike the Y-O peak, 
the intensity of the Y-Y peak decreases from $x=0.025$ to $x=0.1$, as shown in Fig. 9(b). Although the changes in intensity can be explained by various reasons, such as the Debye-Waller factor, coordination number, and amplitude reduction factor, etc, the main reason for this reduction in intensity is most likely originated from the decrease in coordination number. These changes above indicated that the substitution of Mn ions not only affects the nearest neighbor atomic shell of Fe but also affects the nearest neighbor's local structure of $\mathrm{Y}$.

\subsection{Optical measurement}

Figure 10 demonstrates the transmission FT-IR spectra of the pure YFO and $\mathrm{YFM}_{\chi} \mathrm{O}$ powder samples. The ideal perovskite structure belongs to the space group of $\mathrm{O}_{\mathrm{h}}{ }^{1}$ symmetry. Based on group theory, the vibrations in the lattice are consist of $3 F_{1 \rrbracket}+F_{2 \rrbracket}$ modes, of which the $F_{2 \rrbracket}$ is inactive mode [47]. Hence, the perovskite structure should have three absorption bands in IR spectra, which can be attributed to the B-O stretching vibration $\left(F_{1: \mathbb{1} 1}\right)$, the B-O bending vibration $\left(F_{1: \mathbb{2} 2}\right)$, and lattice vibration $\left(F_{1: \mathbb{2} 3}\right)$. The expected energy order is $₫ 1>\nabla 2>\nabla 3$. Any deviation from the cubic symmetry will lead to the splitting of these three bands. At the first sight, our experimental result shows only two $F_{1}$ bands in the IR spectra for all samples. They stem from the modes of $\mathrm{Fe}-\mathrm{O}$ stretching vibration and $\mathrm{O}-\mathrm{Fe}-\mathrm{O}$ bending vibration, respectively, being characteristics of the octahedral $\mathrm{FeO}_{6}$ groups in the perovskite compounds [48]. Based on further observation, it can be seen that the absorption peaks shift to a larger energy side with increasing Mn concentration, which implies the increase of the covalence of the B-O bond and the decrease of the iconicity, as given in Fig. 10(a). A similar phenomenon has been observed by the work of Cao et al and Kumar et al. [23, 49]. In Fig. 10(b), the bands around ca. $1382 \mathrm{~cm}^{-1}$ and $3546 \mathrm{~cm}^{-1}$ are representative of absorption of $\mathrm{NO}^{-3}$ stretching vibrations from the small amount of trapped $\mathrm{NO}^{-3}$ ions in the YFO and -OH from ambient moisture, during the experiment, respectively. However, the intensity of these two bands slightly increases with increasing $\mathrm{Mn}$ content, indicating the incorporation of $\mathrm{Mn}$ ions at Fe-site in YFO. Except for these peaks discussed above, no additional peaks occurred, which indicates the complete substitution of Mn. These are in consistent with the result of XRD and XAFS.

\subsection{Magnetic property}

As is commonly known, the magnetic properties in rare-earth orthoferrites originated from the superexchange interaction of $\mathrm{Fe}^{3+}-\mathrm{O}^{2-}-\mathrm{Fe}^{3+}, \mathrm{R}^{3+}-\mathrm{O}^{2-}-\mathrm{R}^{3+}, \mathrm{R}^{3+}-\mathrm{O}^{2-}-\mathrm{Fe}^{3+}$. In the orthorhombic structure of $\mathrm{YFO}$, there is no magnetic interaction between $\mathrm{Y}^{3+}$ and $\mathrm{Fe}^{3+}$. The $\mathrm{Y}^{3+}$ is diamagnetic as it has fully filled orbitals and each $\mathrm{Fe}^{3+}$ ion is surrounded by six $\mathrm{O}^{2-}$ ions arranged in $\mathrm{FeO}_{6}$ octahedra, and the $\mathrm{O}^{2-}$ is the common apex of the two adjacent octahedral, playing as a $\mathrm{Fe}^{3+}-\mathrm{O}^{2-}-\mathrm{Fe}^{3+}$ super-exchange interaction. The super-exchange interaction is an oxygen mediated exchange between transition metal ions based on virtual hopping processes of the oxygen $2 p$ electrons. The $\mathrm{O}^{2-}$ ions have fully occupied triple degenerate $\left(2 \mathrm{p}_{x}, 2 \mathrm{p}_{y}, 2 \mathrm{p}_{z}\right)$ orbitals with six electrons, while the $5 d$ orbitals of $\mathrm{Fe}^{3+}$ split as triple degenerate $\mathrm{t}_{2 g}\left(\mathrm{~d}_{x y}\right.$, $\left.d_{y z} d_{x z}\right)$ and doubly degenerate $e_{g}\left(d_{x 2-y 2}, d_{z 2}\right)$ orbitals. These $e_{g}$ orbitals of $\mathrm{Fe}^{3+}$ are along the crystal 
axes overlap with the $2 p$ orbitals of $\mathrm{O}^{2-}$, which leads to the super-exchange interaction of $\mathrm{Fe}^{3+}-\mathrm{O}^{2-}-\mathrm{Fe}^{3+}$ through $\mathrm{O}^{2-}$ ions at $180^{\circ} \mathrm{C}$. The GKA (Goodenough, Kanamori, and Anderson) rules were used to predict the super-exchange interaction and the anti-ferromagnetic nature of YFO [50]. However, due to the Dzyaloshinski-Moriya antisymmetric exchange mechanism, each $\mathrm{Fe}^{3+}$ magnetic moment is not being exactly anti-parallel to the moments of the rest of the $\mathrm{Fe}^{3+}$ ions. This leads to the occurrence of weak ferromagnetism in YFO [51]. The schematic representation of the magnetic structure of YFO is presented in Fig. 11(a) and (b), respectively. In this structure, the anti-ferromagnetism is along the a-axis with antiparallel of $\mathrm{Fe}^{3+}$ spin with a small canted angle along the $b$-axis, as shown in Fig. 11(a). Figure 11(b) shows the weak ferromagnetism along the $c$-axis, the canted angle for all $\mathrm{Fe}^{3+}$ spin is parallel arrangement [52]. Figure 12 shows the obvious magnetization hysteresis loops of pure YFO and $\mathrm{YFM}_{x} \mathrm{O}$ samples measured at room temperature. The saturation magnetization $\left(M_{s}\right)$, remnant magnetization $\left(M_{r}\right)$, and coercivity $\left(\mathrm{H}_{\mathrm{c}}\right)$ values of the samples are listed in Table 6 in Fig. 12 for comparison. It is striking to note that all the samples behave as significant hysteresis loops, indicating the ferromagnetic properties of them. When an external field is up to $60 \mathrm{KOe}$, the magnetization has not reached saturation. In this structure, $\mathrm{Y}^{3+}$ is a diamagnetic cation and has a zero magnetic moment, thus, the observed ferromagnetism in our samples could be due to the spin canted of $\mathrm{Fe}^{3+}$ as the source of the magnetic moments [53]. It means that the $\mathrm{Fe}^{3+}$ spins are not completely anti-parallel, but in reality, they may be canted. It is evident from Table 6 that when Mn content increased up to $x=0.075, \mathrm{H}_{c}$ decreased while $M_{S}$ and $M_{r}$ increased compared to those of YFO. A sudden decrease of $M_{s}$ and $M_{r}$ values occurs for $M n$ content at $x=0.1$. This phenomenon may be due to the lattice shrinking, resulting in enhanced internal stress with the increase of Mn substitution. From Fig. 12(a), from left to right, it can be seen that the magnetization of the samples strongly depends on the Mn concentration. Hysteretic loops of $\mathrm{YFM}_{x} \mathrm{O}$ have been narrowing with increasing Mn concentration, which reveals that the magnetic property changes from ferromagnetic to paramagnetic behavior. It can be accounted for several reasons as follows. First, it could be explained by the paramagnetic component and weak ferromagnetic component. Secondly, it could be due to the $\mathrm{Fe}^{3+}-\mathrm{O}^{2-}-\mathrm{Fe}^{3+}$ super-exchange interaction, which is the dominated magnetic interaction in the $\mathrm{YFM}_{x} \mathrm{O}$ system and may induce the weaker ferromagnetism. The $\mathrm{Fe}^{3+}-\mathrm{O}^{2-}-$ $\mathrm{Fe}^{3+}$ super-exchange effect becomes stronger due to lattice change caused by different radius between $\mathrm{Mn}^{3+}$ and $\mathrm{Fe}^{3+}$ ions. Besides, the paramagnetic contribution from the $\mathrm{Mn}^{3+}$ ions cannot be ignored. $\mathrm{A}$ similar paramagnetic component caused by rare-earth ions has also been found in $\mathrm{Bi}_{1}-\mathrm{Gd}_{x} \mathrm{FeO}_{3}$ [54] and $\mathrm{Y}_{1-x} \mathrm{Gd}_{x} \mathrm{FeO}_{3}$ [55]. The higher $\mathrm{Mn}$ concentration, the weaker ferromagnetic property, which is consistent with the change of the hysteretic loop.

\section{Conclusions}

In this work, the effect of Fe-site $\mathrm{Mn}^{3+}$ doping with $0 \leq x \leq 0.1$ concentration on structural, morphological, local electrical, optical and magnetic properties of $\mathrm{YFeO}_{3}$ powders synthesized by using the hydrothermal method has been investigated. In the XRD patterns, the sharp and well-defined peaks show that all 
samples have an orthorhombic structure with space group Pnma. By using Rietveld fitting of the XRD profile we could confirm the orthorhombic crystalline structure of $\mathrm{YFeO}_{3}$. Refined structure of $x=0$ and $x$ $=0.1$ samples also reveal the decreasing parameters with increasing Mn content, which was considered to be the smaller ionic radii of $\mathrm{Mn}^{3+}$ compared with that of $\mathrm{Fe}^{3+}$. As shown by SEM images, with increasing dopant concentration, the layered shape changes to a multilayered shape and a large reduction of particle size observed when $x=0.1$ with larger agglomerates shape. XAFS spectroscopy, including XANES and EXAFS has been used to investigate and obtain the structural information around $F e$ and $Y$ atoms in $\mathrm{YFM}_{X} \mathrm{O}$ samples. However, the spectra have a similar shape for the whole series. The results of XANES confirm the 3 + oxidation states of $Y$ and $F$ ions in the $Y F M_{X} O$ powders. Furthermore, the results from the EXAFS indicate that substitution of $\mathrm{Mn}$ ions not only affects the nearest neighbor atomic shell of Fe but also affects the nearest neighbor's local structure of Y. The FT-IR spectra reveal the characteristic vibrations of the obtained $\mathrm{YFM}_{x} \mathrm{O}$ samples. The magnetization of the $\mathrm{YFM}{ }_{x} \mathrm{O}$ samples has a close relationship with the $\mathrm{Mn}$ concentration. Magnetic hysteresis loop measurement reveals that the magnetic property changes from ferromagnetic to paramagnetic behavior with increasing $\mathrm{Mn}$ concentrations. Our results suggest that the $\mathrm{Mn}^{3+}$ ions play an important role to the local structural and magnetic properties of $\mathrm{YFeO}_{3}$. Furthermore, the present work promotes the need for better understanding of the local electronic structure in the $\mathrm{YFeO}_{3}$.

\section{Declarations}

\section{Acknowledgments}

The authors would like to acknowledge the financial supported by the graduate research fund offered by Xiamen University. The authors report no declarations of interest.

\section{References}

1. Kimura T, Goto. T, Shintani. H et al (2003) Magnetic control of ferroelectric polarization. Nature 426:55-58

2. Brown C (1975) S. Magnetic oxides parts 1 and 2. Phys Bull 26:546-547

3. White RL (1969) Review of recent work on the magnetic and spectroscopic properties of the rareearth orthoferrites. J Appl Phys 40:1061-1069

4. Didosyan Y, S, Hauser H (1998) Observation of Bloch lines in yttrium orthoferrite. Phys Lett $A$ 238:395-397

5. Didosyan Y, S, Hauser. H, Fiala. W et al (2002) Fast latching type optical switch. J Appl Phys 91:7000-7002

6. Wang K, F LJM, Ren. (2009) Z. F. Multiferroicity: the coupling between magnetic and polarization orders. Adv Phys 58:321-448 
7. Bamzai K, K, Bhat M (2015) Electrical and magnetic properties of some rare earth orthoferrites $\left(\mathrm{RFeO}_{3}\right.$ where $\left.\mathrm{R}=\mathrm{Y}, \mathrm{Ho}, \mathrm{Er}\right)$ systems. Integr Ferroelectr 158:108-122

8. Bombik. A, Leśniewska. B, Mayer. J et al (2003) Crystal structure of solid solutions $\mathrm{REFe}_{1-x}(\mathrm{Al}$ or $\mathrm{Ga})_{x} \mathrm{O}_{3}(\mathrm{RE}=\mathrm{Tb}, \mathrm{Er}, \mathrm{Tm})$ and the correlation between superexchange interaction $\mathrm{Fe}^{+3}-\mathrm{O}^{-2}-\mathrm{Fe}^{+3}$ linkage angles and Néel temperature. J Magn Magn Mater 257:206-219

9. Zhou Z, Q, Guo. L, Yang H, X et al (2014) Hydrothermal synthesis and magnetic properties of multiferroic rare-earth orthoferrites. J Alloy Compd 583:21-31

10. Coutinho P, V, Cunha. F, Barrozo P (2017) Structural, vibrational and magnetic properties of the

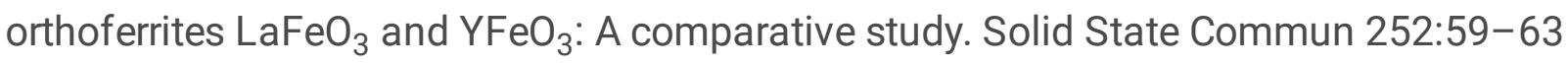

11. Dho J (2005) Blamire. M. G. Competing functionality in multiferroic $\mathrm{YMnO}_{3}$. Appl Phys Lett 87:252504

12. Rosales-González O, Sánchez-De Jesús F, Cortés-Escobedo CA et al (2018) Crystal structure and multiferroic behavior of perovskite $\mathrm{YFeO}_{3}$. Ceram Int 44:15298-15303

13. Geller S (1956) Crystal structure of gadolinium orthoferrite, $\mathrm{GdFeO}_{3}$. J Chem Phys 24:1236-1239

14. Jin ZM, Mics. Z, Ma G, H et al (2013) Single-pulse terahertz coherent control of spin resonance in the canted antiferromagnet $\mathrm{YFeO}_{3}$, mediated by dielectric anisotropy. Phys Rev B 87:269-275

15. Kim T, H, Sun Y, H HJW et al (2014) Coherently controlled spin precession in canted antiferromagnetic $\mathrm{YFeO}_{3}$ using terahertz magnetic field. Appl Phys Express 7:093007

16. Cho K, Hur S, Park S (2017) The absence of ferroelectricity and the origin of depolarization currents in $\mathrm{YFe}_{0.8} \mathrm{Mn}_{0.2} \mathrm{O}_{3}$. Appl Phys Lett 110:162905

17. Xie T, Shen. H, Wu. A et al (2016) Crystal growth, spin reorientation and magnetic anisotropy of $\mathrm{YFe}_{0.8} \mathrm{Mn}_{0.2} \mathrm{O}_{3}$ single crystal. Solid State Commun 247:64-67

18. Xie T, Shen. $H$, Zhao. X et al (2016) Single crystal growth, magnetic and thermal properties of perovskite $\mathrm{YFe}_{0.6} \mathrm{Mn}_{0.4} \mathrm{O}_{3}$ single crystal. J Magn Magn Mater 417:143-147

19. Wang. ZC, Wang. X (2016) Z, et al. Dielectric relaxation, electric modulus and ac conductivity of Mndoped $\mathrm{YFeO}_{3}$. Ceram Int 42:19461-19465

20. Wang. ZC, Z, Yan. $\mathrm{H}$ et al (2014) Structural and magnetic properties in $\mathrm{YFe}_{0.8} \mathrm{Mn}_{0.2} \mathrm{O}_{3}$ ceramics. Mater Lett 136:15-17

21. Deka B, Ravi. S, Perumal. A et al (2017) Effect of Mn doping on magnetic and dielectric properties of $\mathrm{YFeO}_{3}$. Ceram Int 43:1323-1334

22. Deka B, Ravi. S, Perumal A (2016) Study of Exchange Bias in Mn-Doped $\mathrm{YFeO}_{3}$ Compound. J Supercon Nov Magn 29:2165-2170

23. Cao X, Q, Kim CS (2010) Yoo. H. I. Effect of Substitution of Manganese for Iron on the Structure and Electrical Properties of Yttrium Ferrite. J Am Ceram Soc 84:1265-1272

24. VA, Noudem. J G, Fourrez. S et al (2006) The influence of iron substitution to manganese on the physical properties of $\mathrm{YMnO}_{3}$. Solid State Sci 8:137-141 
25. Shen. H, Xu. J, Jin. M et al (2012) Influence of manganese on the structure and magnetic properties of $\mathrm{YFeO}_{3}$ nanocrystal. Ceram Int 38:1473-1477

26. Mandal P, Sundarayya. BVS (2011) Y, et al. Spin-reorientation, ferroelectricity, and magnetodielectric effect in $\mathrm{YFe}_{1}-{ }_{x} \mathrm{Mn}_{x} \mathrm{O}_{3}(0.1 \leq x \leq 0.40)$. Phy Rev Lett 107:137202

27. Zaghrioui. M, Grenèche. JM, Autret-Lambert $C$ et al (2011) Effect of Fe substitution on multiferroic hexagonal $\mathrm{YMnO}_{3}$. J Magn Magn Mater 323:509-514

28. Zhang. K, Bunker. G, B, Zhang. G et al (1988) Extended x-ray-absorption fine-structure experiment on the high $-T_{\mathrm{C}}$ superconductor $\mathrm{YBa}_{2} \mathrm{Cu}_{7-\delta}$. Phys Rev B 37:3375-3380

29. Moreno S, D, Chaboy J (2009) Ab initio X-ray absorption spectroscopy study of the solvation structure of yttrium (III) in dimethyl sulfoxide. J Phys Chem B 113:3527-3535

30. Sayers D, E, Stern E, A (1975) Lytle. F. W. New Method to Measure Structural Disorder: Application to $\mathrm{GeO}_{2}$ Glass*. Phys Rev Lett 35:584-587

31. Ravel B, Newville. M, ATHENA (2005) ARTEMIS, HEPHAESTUS: data analysis for X-ray absorption spectroscopy using IFEFFIT. J Synchrotron Rad 12:537-541

32. Pedro- GF, Sanchez-De Jesús F, Cortes-Escobedo CA et al (2017) Mechanically assisted synthesis of multiferroic $\mathrm{BiFeO}_{3}$ : Effect of synthesis parameters. J Alloy Compd 711:77-84

33. Racu A, V, Ursu D, H, Kuliukova O, V et al (2015) Direct low temperature hydrothermal synthesis of $\mathrm{YFeO}_{3}$ microcrystals. Mater Lett 140:107-110

34. Boulay D, D, Maslen E, N (1995) Streltsov. V. A. A synchrotron X-ray study of the electron density in $\mathrm{YFeO}_{3}$. Acta Crystallogr B 51:921-929

35. Liu. WJ, Bai. Y (2016) X, et al. Crystal structure, leakage conduction mechanism evolution and enhanced multiferroic properties in $\mathrm{Y}$-doped $\mathrm{BiFeO}_{3}$ ceramics. Ceram Int 42:13395-13403

36. Phokha S, Pinitsoontorn S, Maensiri S (2012) Room-temperature ferromagnetism in Co-doped $\mathrm{CeO}_{2}$ nanospheres prepared by the polyvinylpyrrolidone-assisted hydrothermal method. J Appl Phys 112:113904

37. Zhou Z, Q, Guo. L, Yang H, X et al (2014) Hydrothermal synthesis and magnetic properties of multiferroic rare-earth orthoferrites. J Alloy Compd 583:21-31

38. Geng F, X, Matsushita. Y, Ma. R, Z et al. Synthesis and properties of well-crystallized layered rare-earth hydroxide nitrates from homogeneous precipitation. Inorg Chem 2010, 48: 6724-6730

39. Gholam T, Ablat. A, Mamat. M et al (2017) An experimental study of the local electronic structure of B-site gallium doped bismuth ferrite powders. Phys Lett A 381:2367-2373

40. White E, W (1966) Mckinstry. H. A. Chemical effect on x-ray absorption-edge fine structure. In: Mallett GR, Fay MJ, Mueller WM (eds) Advances in X-Ray Analysis. Springer, Boston, pp 376-392

41. Aznar. B BJ, Garcia. J et al (2008) Charge disproportionation in $\mathrm{La}_{1}-{ }_{x} \mathrm{Sr}_{x} \mathrm{FeO}_{3}$ probed by diffraction and spectroscopic experiments. Phys Rev B 77:054107 
42. Li. Y, Zhang. H, Liu. H et al (2014) Magnetic properties and local structure of the binary elements codoped $\mathrm{Bi}_{1}-{ }_{x} \mathrm{La}_{x} \mathrm{Fe}_{0.95} \mathrm{Mn}_{0.05} \mathrm{O}_{3}$. J Alloy Compd 592:19-23

43. Seremak-Peczkis P, Schneider. K, Zajączkowski. W et al (2009) XAFS study of $\mathrm{BaCe}_{1-x} \mathrm{Ti}_{x} \mathrm{O}_{3}$ and $\mathrm{Ba}_{1}$ $-{ }_{y} \mathrm{Ce}_{1}-{ }_{x} \mathrm{Y}_{x} \mathrm{O}_{3}$ protonic solid electrolytes. Radiat Phys Chem 78:S86-S88

44. Yotburut B, Yamwong. T, Thongbai. P et al (2014) Synthesis and characterization of coprecipitationprepared $\mathrm{La}$-doped $\mathrm{BiFeO}_{3}$ nanopowders and their bulk dielectric properties. Jpn J Appl Phys 53:06JG13

45. Roe A, L, Schneider DJ (1984) Mayer. R. J, et al. X-ray Absorption Spectroscopy of Iron-Tyrosinate Proteins. J Am Chem Soc 106:1676-1681

46. Finck N, Bouby. M, Dardenne. K et al (2017) Yttrium co-precipitation with smectite: A polarized XAS and AsFIFFF study. Appl Clay Sci 137:11-21

47. Yue W, U, Zuolong Y, U, Liu S (1994) Preparation, crystal structure, and vibrational spectra of perovskite-type mixed oxides $\mathrm{LaM}_{y} \mathrm{M}_{1-y}^{\prime} \mathrm{O}_{3}\left(\mathrm{M}, \mathrm{M}^{\prime}=\mathrm{Mn}\right.$, Fe, Co). J Solid State Chem 112:157-160

48. Zaidi FS (2009) M. Bismuth ferrite $\left(\mathrm{BiFeO}_{3}\right)$ nanopowder prepared by sucrose-assisted combustion method: A novel and reusable heterogeneous catalyst for acetylation of amines, alcohols and phenols under solvent-free conditions. J Mol Catal A-Chem 299:18-25

49. Kumar M, Sati P, C, Chhoker. S et al (2015) Electron spin resonance studies and improved magnetic properties of $\mathrm{Gd}$ substituted $\mathrm{BiFeO}_{3}$ ceramics. Ceram Int 41:777-786

50. Anderson P (1959) W. New approach to the theory of superexchange interactions. Phys Rev 115:213

51. Ahmad T, Lone I, H, Ansari S, G et al (2017) Multifunctional properties and applications of yttrium ferrite nanoparticles prepared by citrate precursor route. Mater Design 126:331-338

52. Hahn S, E, Podlesnyak A, A, Ehlers G et al (2014) Inelastic neutron scattering studies of $\mathrm{YFeO}_{3}$. Phys Rev B 89:014420

53. Wang M, Wang. T, Song SH et al (2017) Effect of calcination temperature on structural, magnetic and optical properties of multiferroic $\mathrm{YFeO}_{3}$ nanopowders synthesized by a low temperature solid-state reaction. Ceram Int 43:10270-10276

54. Hu W, W, Chen. Y, Yuan HM et al (2011) Structure, Magnetic, and Ferroelectric Properties of $\mathrm{Bi}_{1}$ ${ }_{x} \mathrm{Gd}_{x} \mathrm{FeO}_{3}$ Nanoparticles. J Phys Chem C 115:8869-8875

55. Yuan. X, Sun. Y, Xu M (2012) Effect of Gd substitution on the structure and magnetic properties of $\mathrm{YFeO}_{3}$ ceramics. J Solid State Chem 196:362-366

\section{Figures}




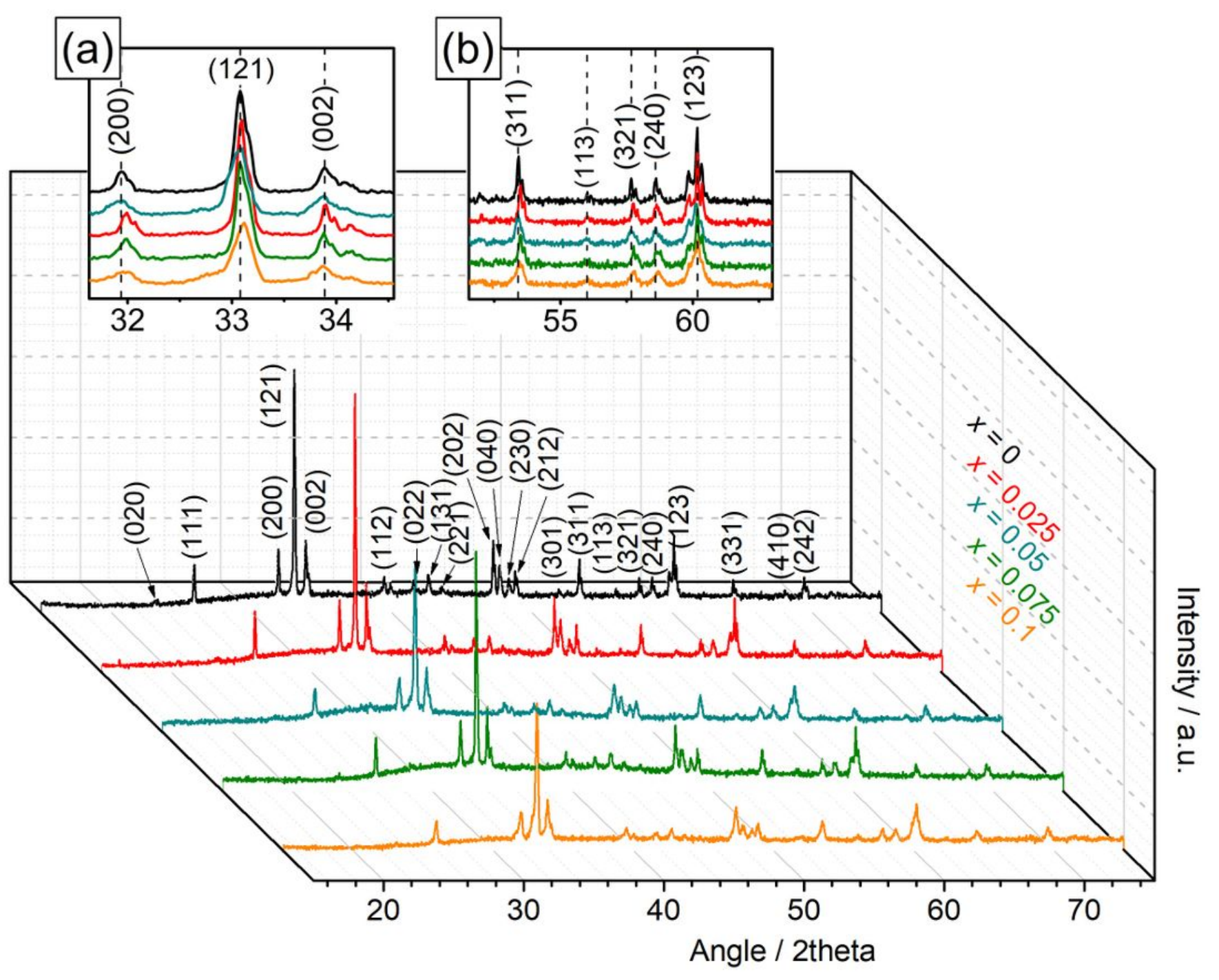

Figure 1

XRD patterns of YFMxO with $0 \leq x \leq 0.1$. (a)-(b) Selected part of XRD patterns. The data were offset for clarity. 


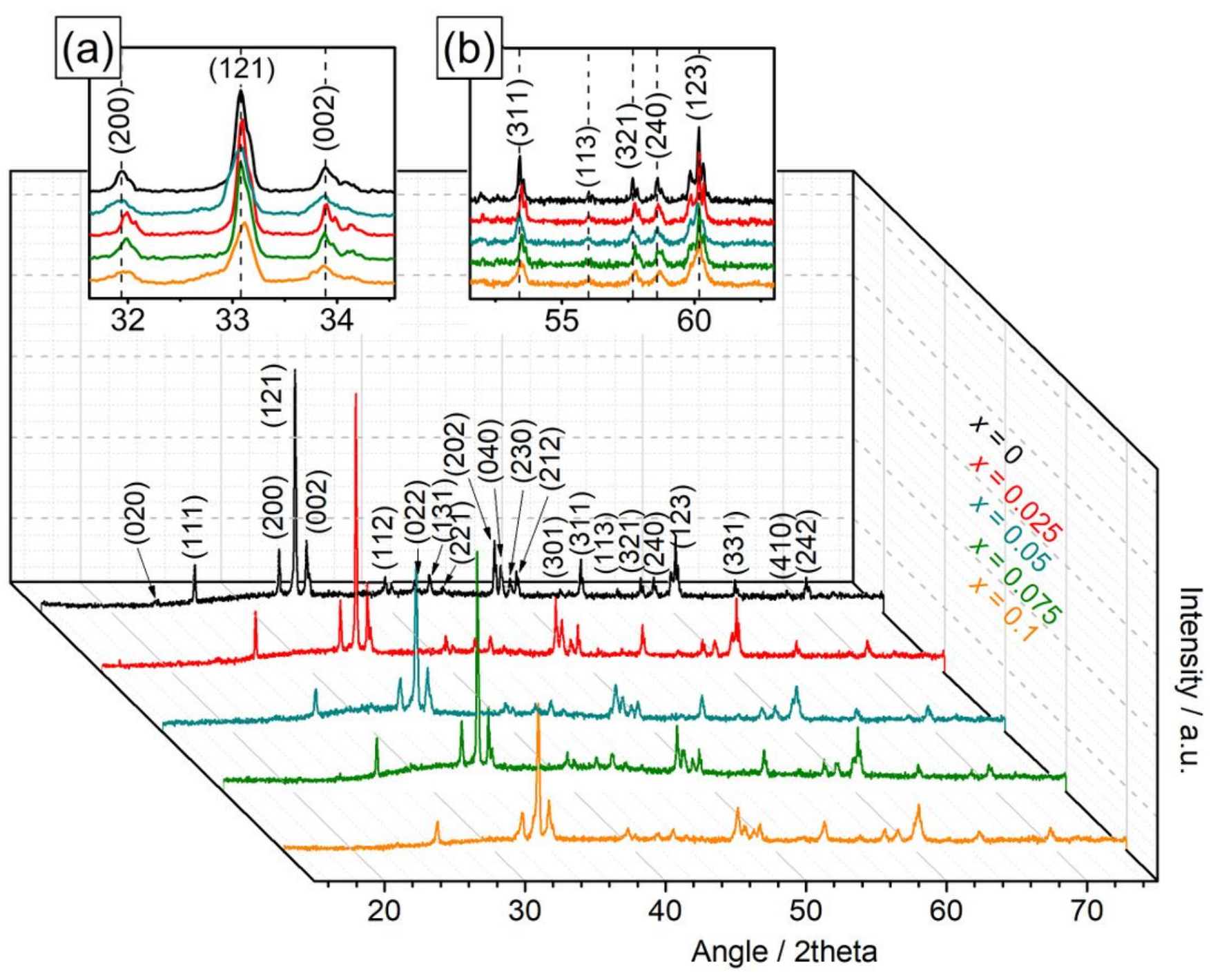

Figure 1

XRD patterns of YFMXO with $0 \leq x \leq 0.1$. (a)-(b) Selected part of XRD patterns. The data were offset for clarity. 


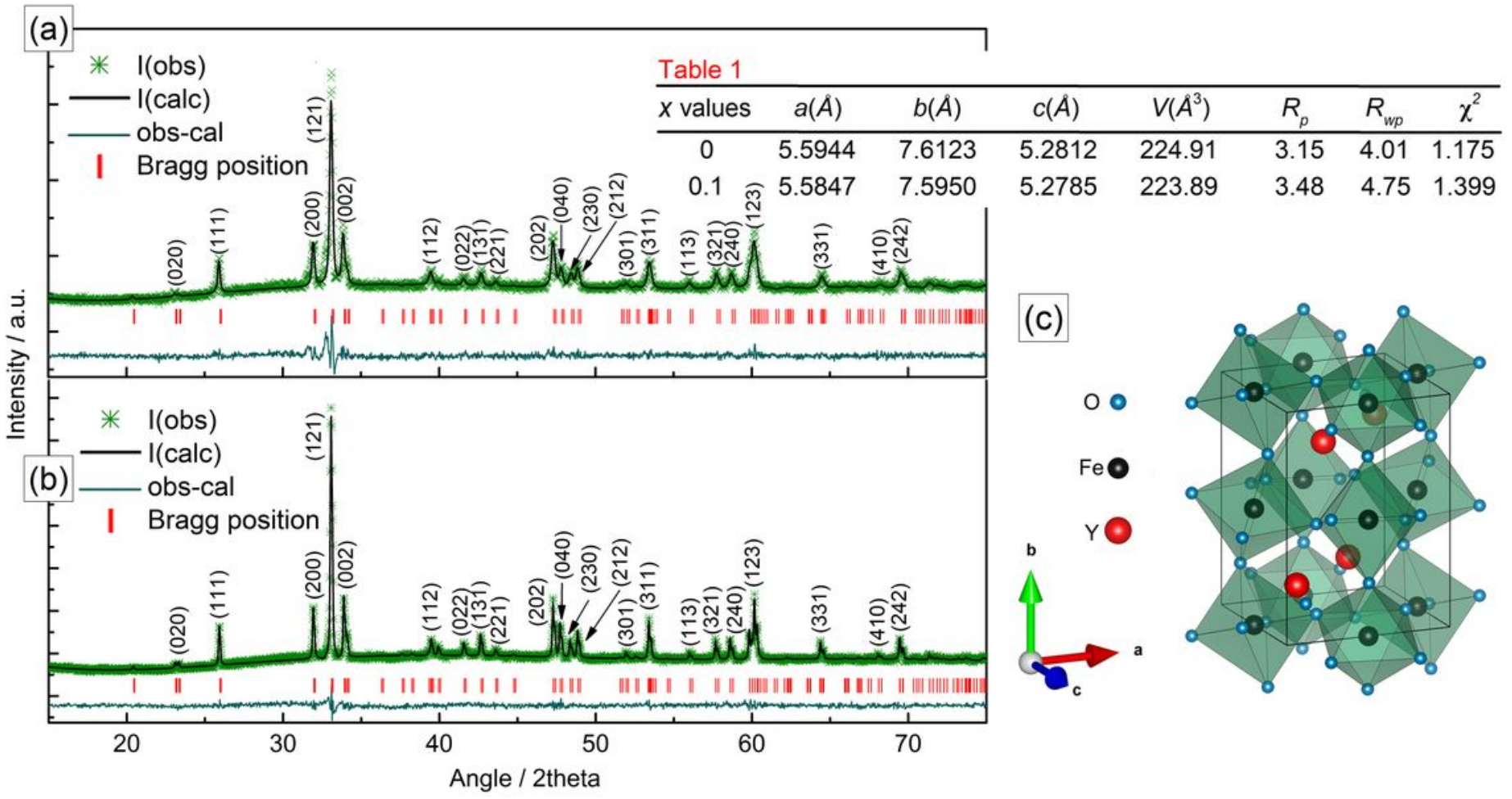

Figure 2

Rietveld refinement analyzed the diffractogram of YFMxO. (a) $x=0$ (b) $x=0.1$ (c) Schematic presentation of the YFO orthorhombic Pnma unit cell. The lattice parameters of YFMxO with $x=0$ and $x=0.1$ were listed in Table 1.

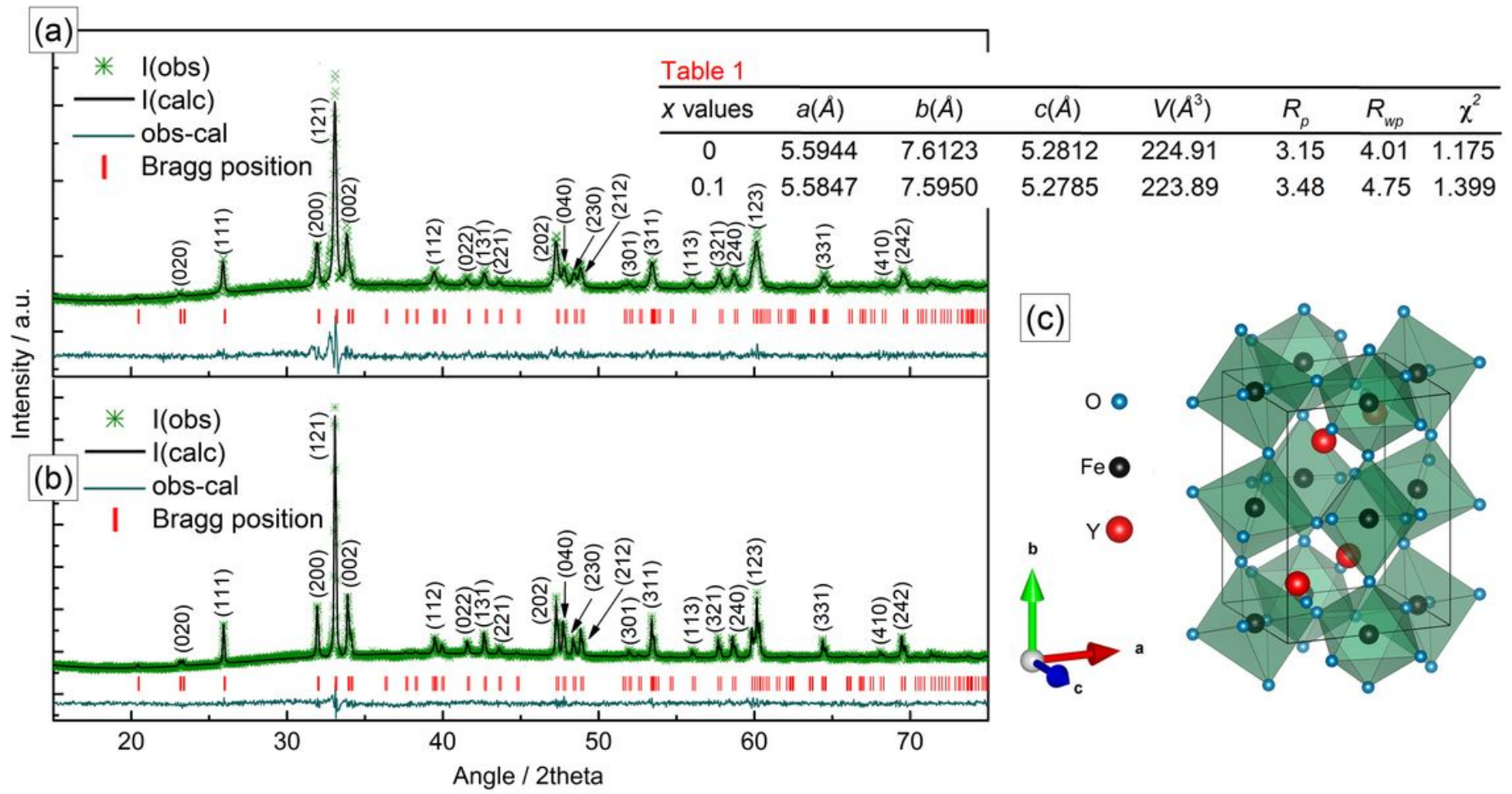

Figure 2 
Rietveld refinement analyzed the diffractogram of YFMxO. (a) $x=0$ (b) $x=0.1$ (c) Schematic presentation of the YFO orthorhombic Pnma unit cell. The lattice parameters of YFMxO with $x=0$ and $x=0.1$ were listed in Table 1.
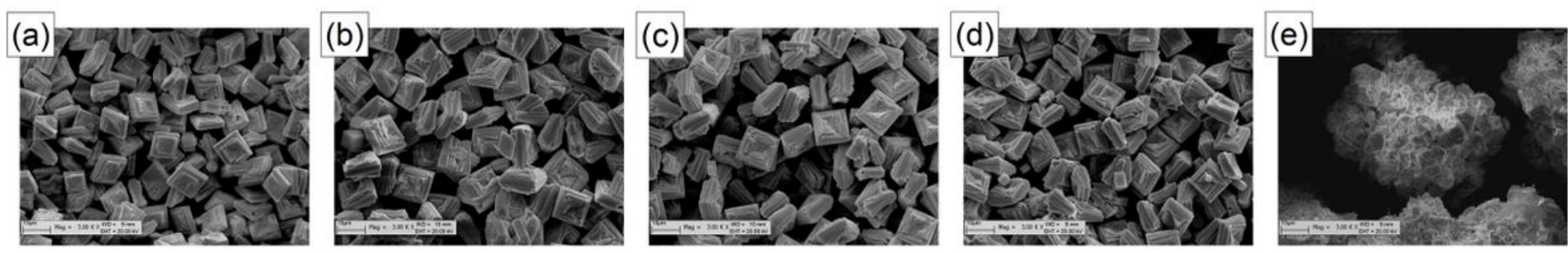

(f)
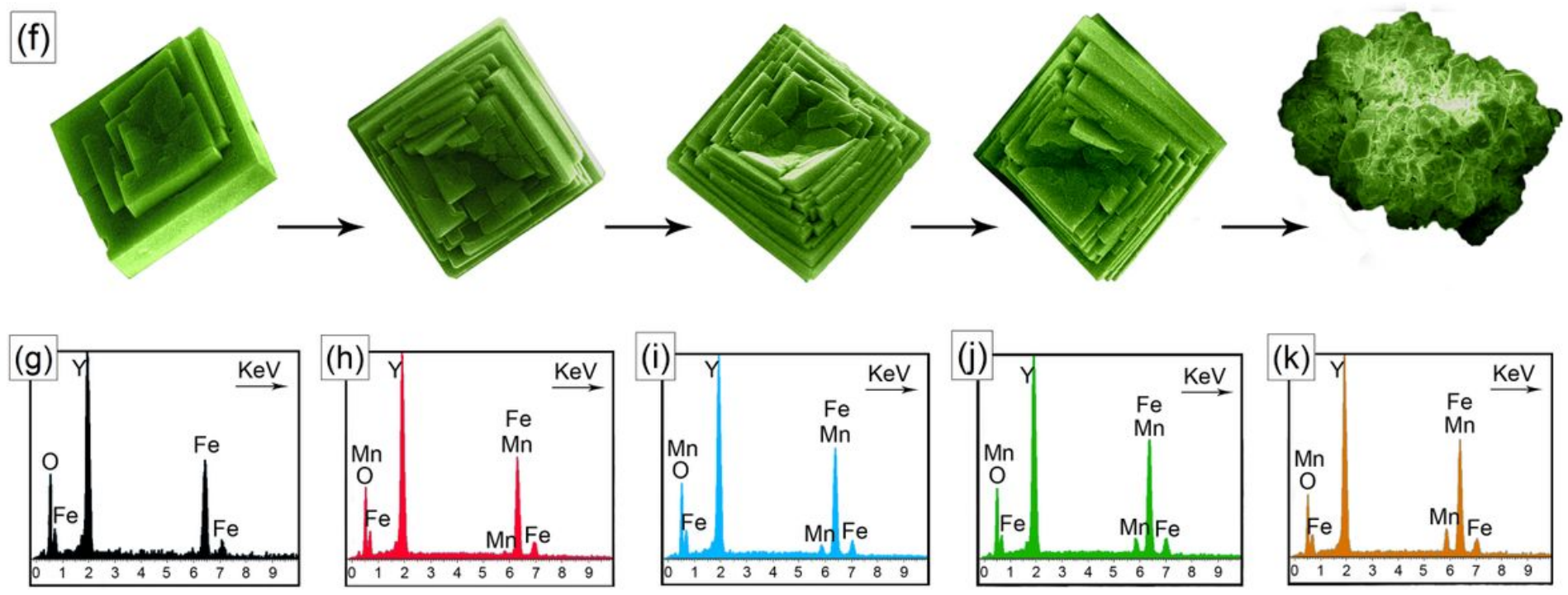

\section{Figure 3}

SEM graphs of YFMxO. (a) $x=0$ (b) $x=0.025$ (c) $x=0.05$ (d) $x=0.075$ (e) $x=0.1$. (f) Schematic illustration of shape evolution. The color of all images was changed to green for clarity. EDS patterns of YFMxO. (g) $x=0$ (h) $x=0.025$ (i) $x=0.05(j) x=0.075(k) x=0.1$. 

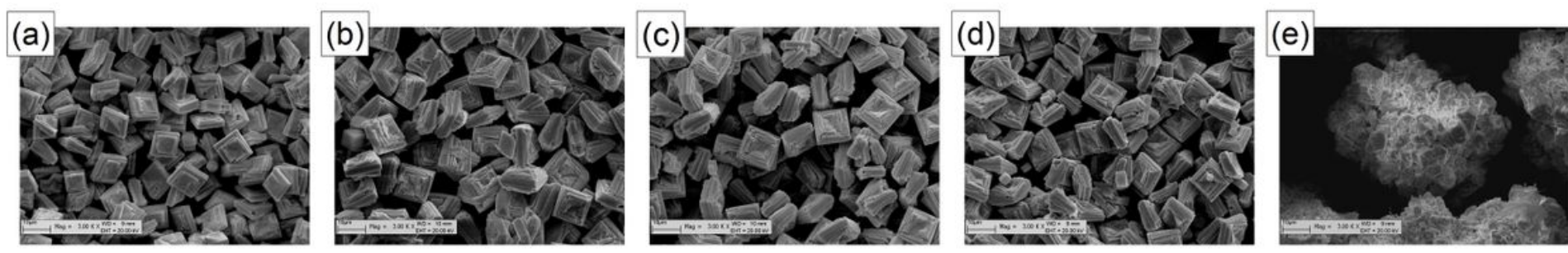

(f)
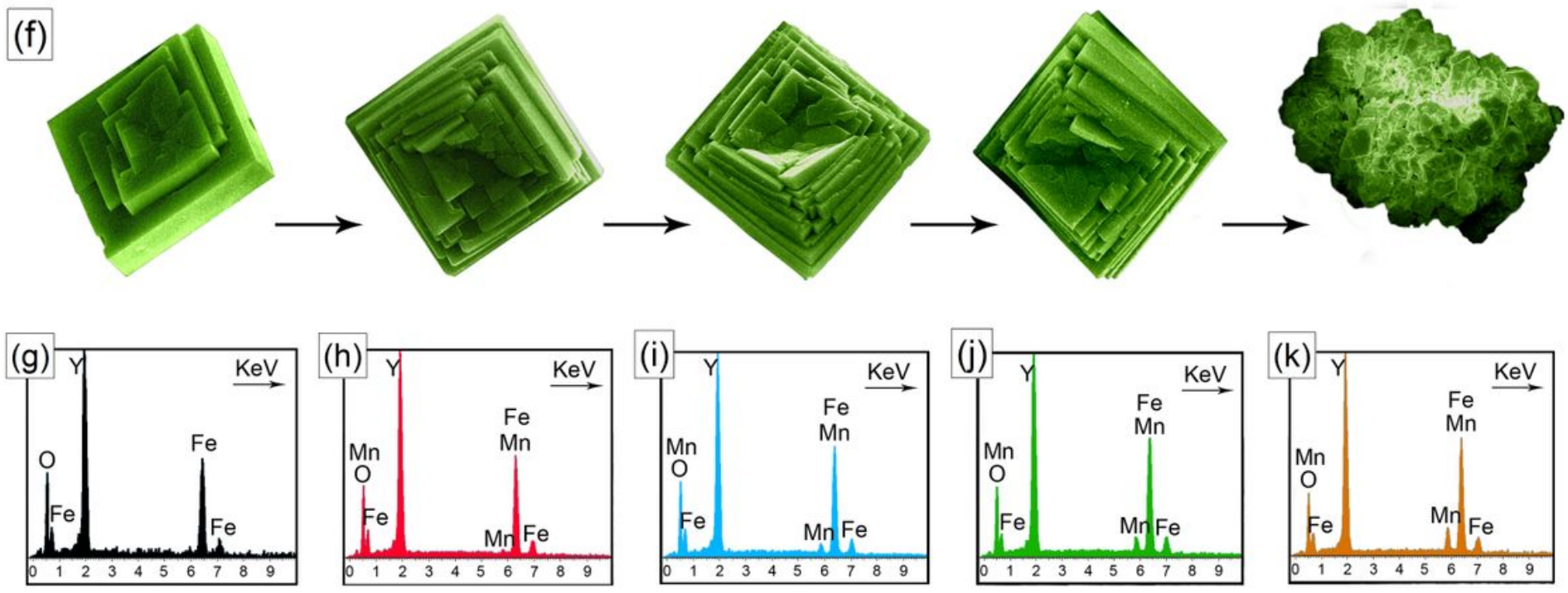

Figure 3

SEM graphs of YFMxO. (a) $x=0$ (b) $x=0.025$ (c) $x=0.05$ (d) $x=0.075$ (e) $x=0.1$. (f) Schematic illustration of shape evolution. The color of all images was changed to green for clarity. EDS patterns of YFMxO. (g) $x=0$ (h) $x=0.025$ (i) $x=0.05(j) x=0.075(k) x=0.1$. 


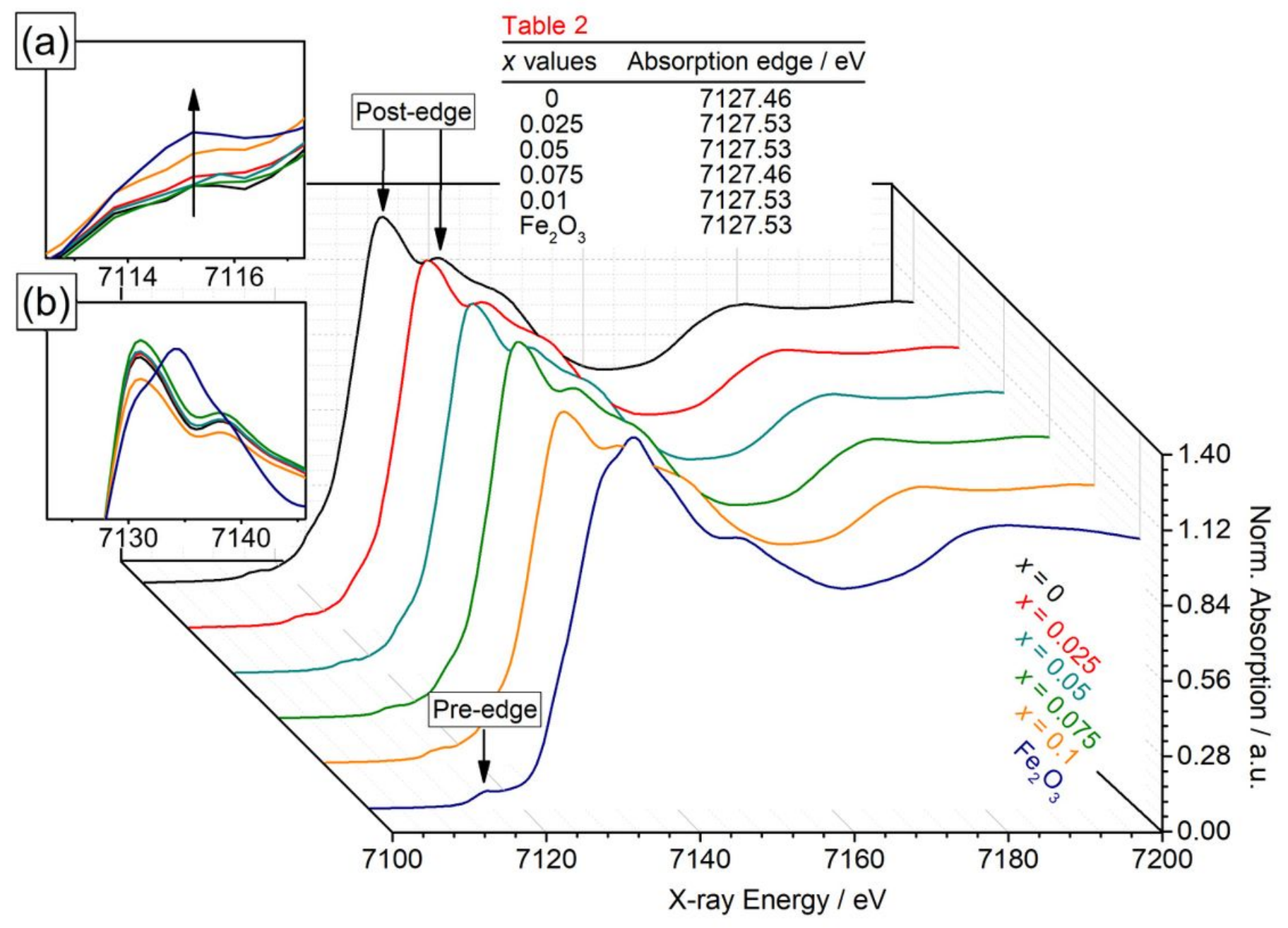

Figure 4

Fe K-edge XANES spectra of YFMxO with $0 \leq x \leq 0.1$ and reference Fe2O3. (a) Enlarged XANES spectrum. The data were offset for clarity. The absorption energies were listed in Table 2. 


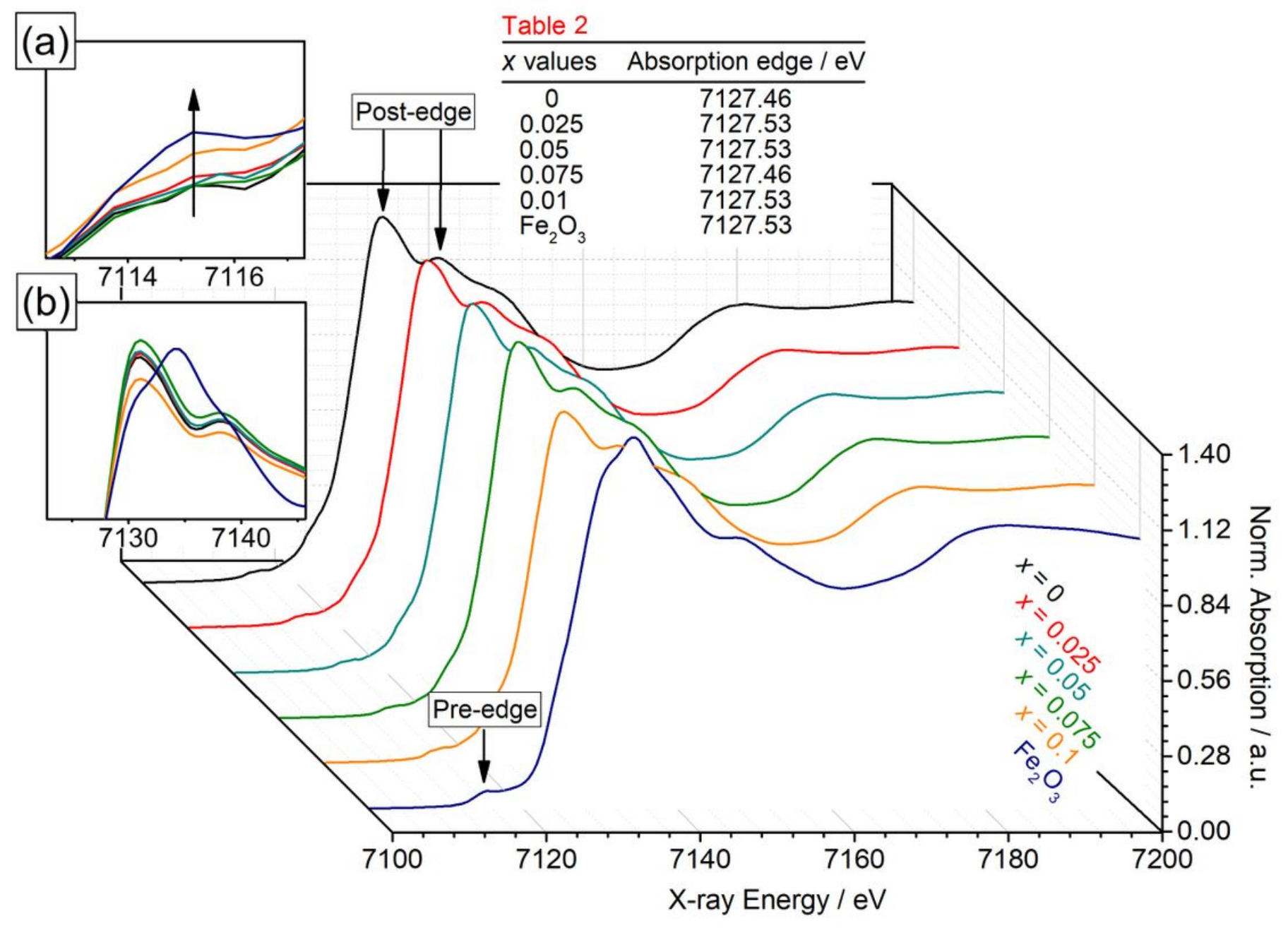

Figure 4

Fe K-edge XANES spectra of YFMxO with $0 \leq x \leq 0.1$ and reference Fe2O3. (a) Enlarged XANES spectrum. The data were offset for clarity. The absorption energies were listed in Table 2. 


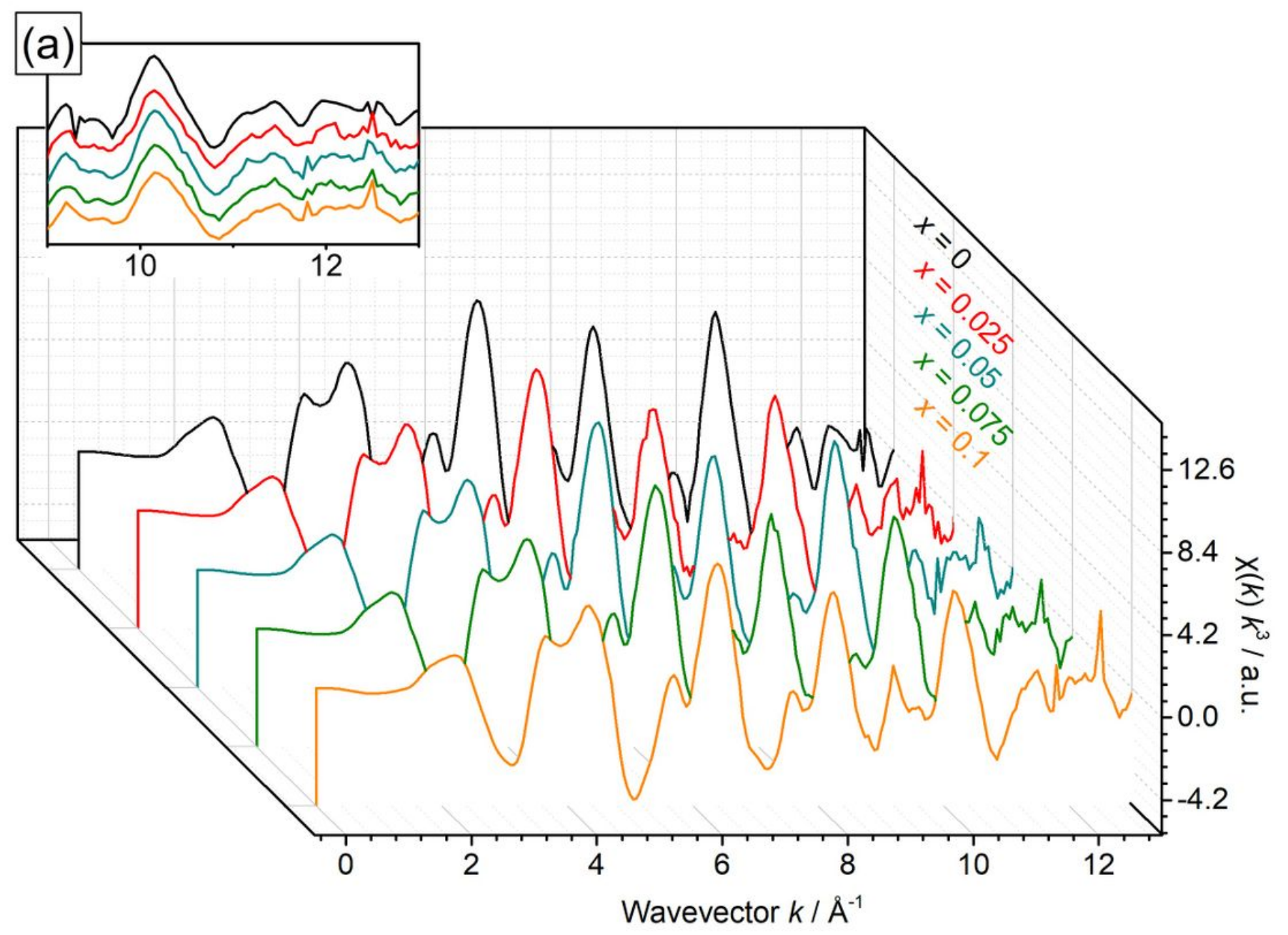

Figure 5

Fe K-edge EXAFS oscillations of YFMxO with $0 \leq x \leq 0.1$. (a) Selected part of the EXAFS spectrum. The data were offset for clarity. 


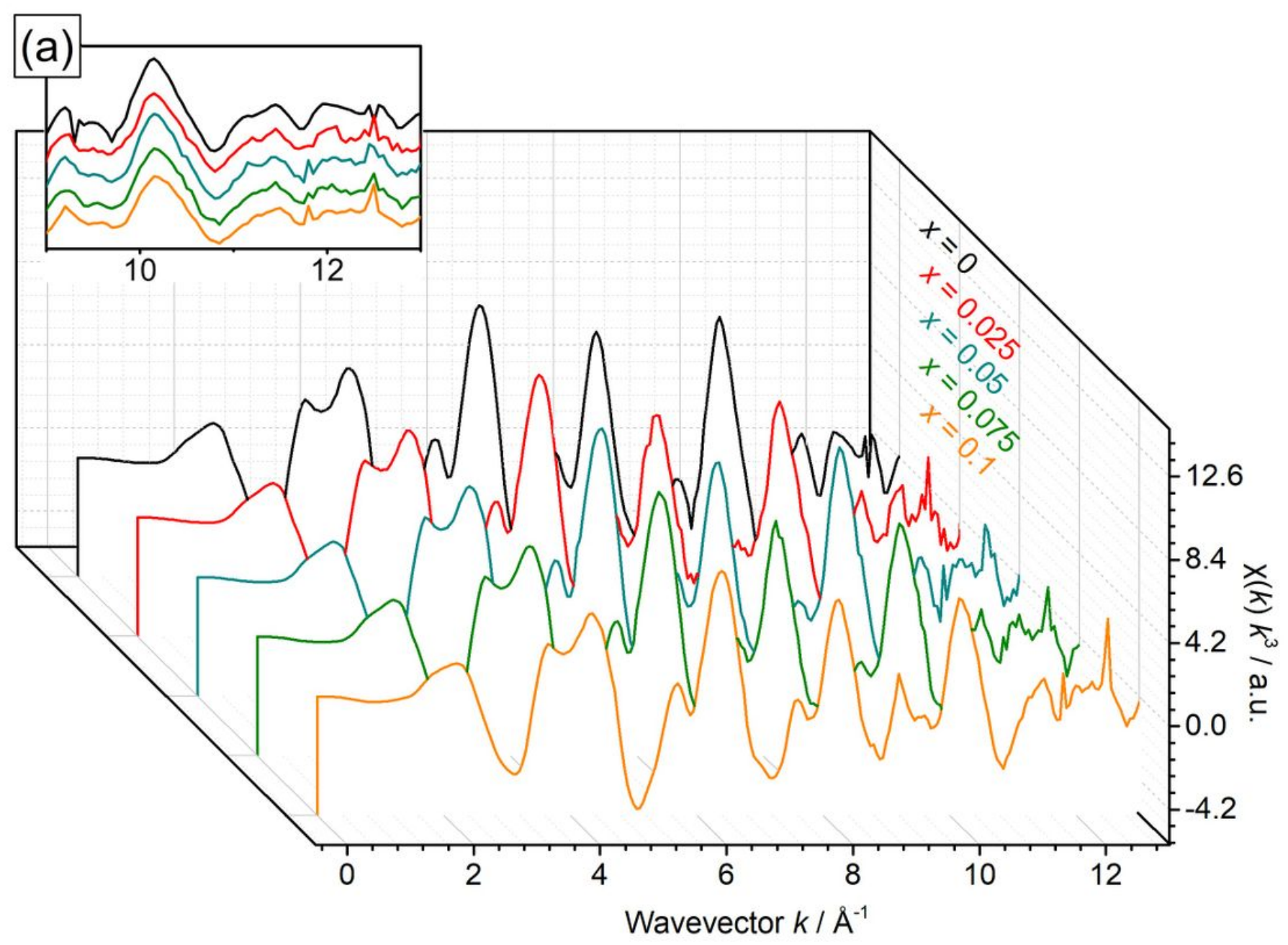

Figure 5

Fe K-edge EXAFS oscillations of YFMxO with $0 \leq x \leq 0.1$. (a) Selected part of the EXAFS spectrum. The data were offset for clarity. 


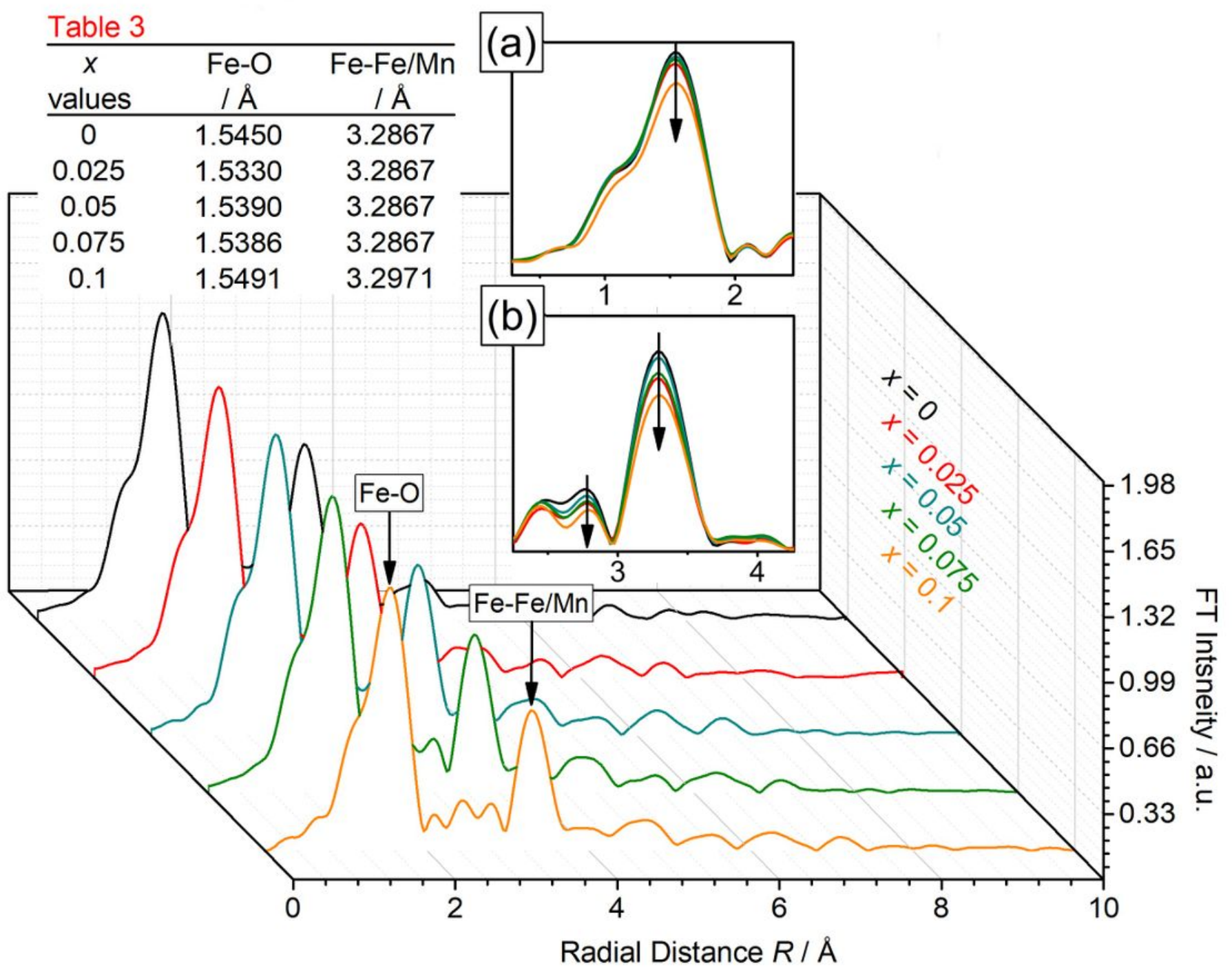

Figure 6

Fourier transform of Fe K-edge EXAFS functions of YFMxO with $0 \leq x \leq 0.1$. (a) Magnified EXAFS spectrum. The data were offset for clarity. The $\mathrm{Fe}-\mathrm{O}$ and $\mathrm{Fe}-\mathrm{Fe} / \mathrm{Mn}$ interatomic distances were listed in Table 3. 


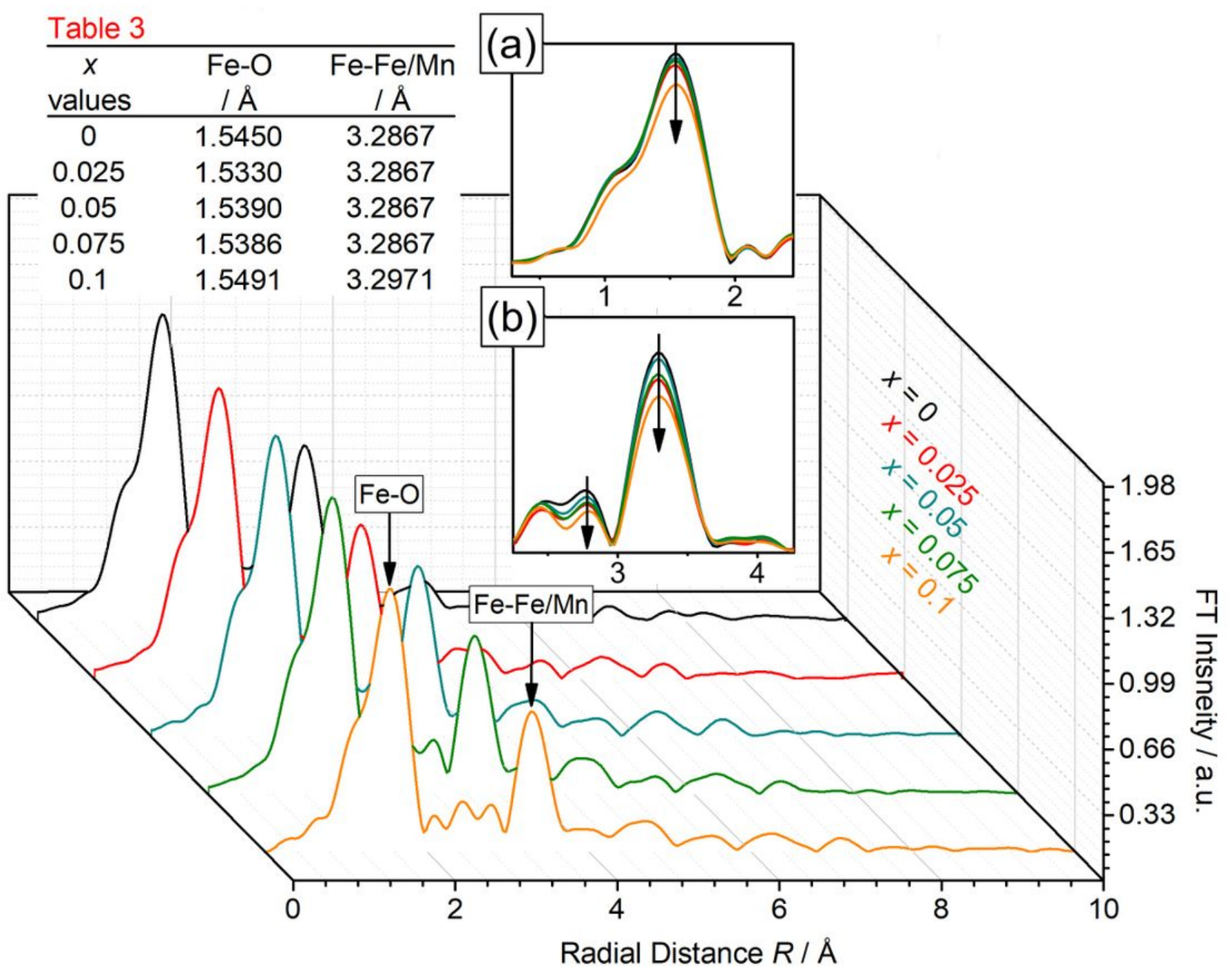

Figure 6

Fourier transform of Fe K-edge EXAFS functions of YFMxO with $0 \leq x \leq 0.1$. (a) Magnified EXAFS spectrum. The data were offset for clarity. The $\mathrm{Fe}-\mathrm{O}$ and $\mathrm{Fe}-\mathrm{Fe} / \mathrm{Mn}$ interatomic distances were listed in Table 3. 


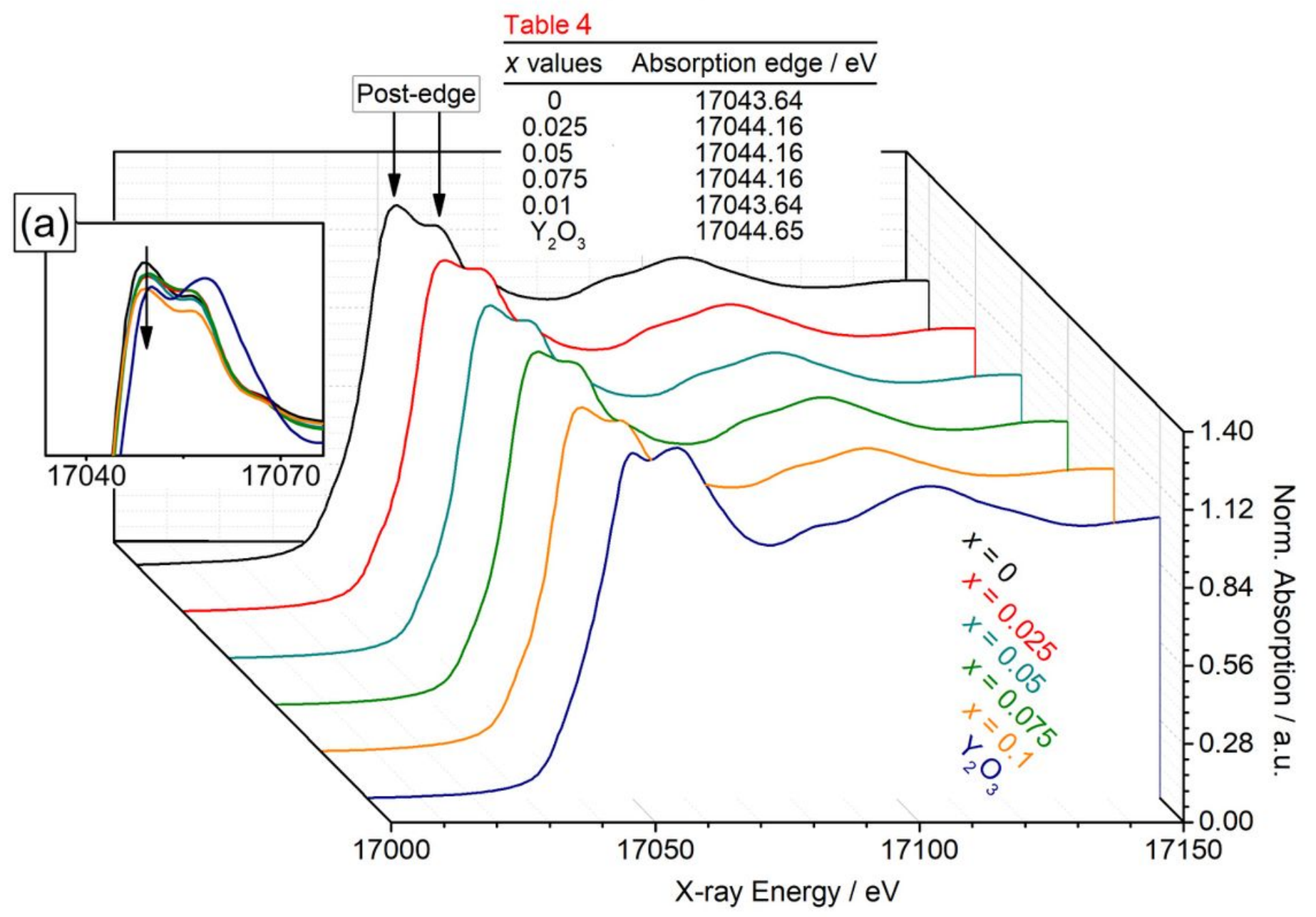

Figure 7

Y K-edge XANES spectra of YFMxO with $0 \leq x \leq 0.1$ and reference $Y 203$. (a) Enlarged XANES spectrum. The data were offset for clarity. The absorption energies were listed in Table 4. 


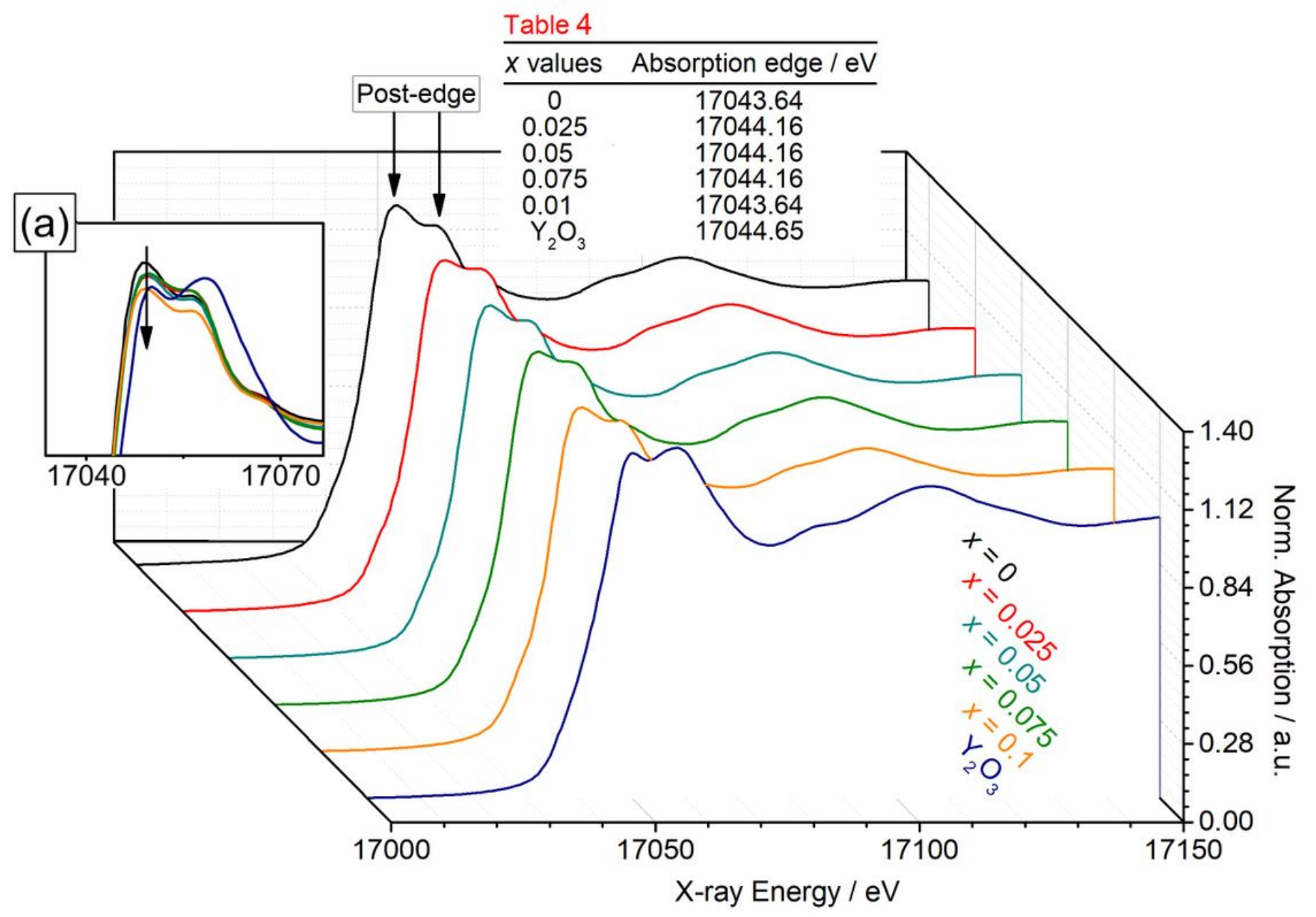

Figure 7

Y K-edge XANES spectra of YFMxO with $0 \leq x \leq 0.1$ and reference $Y 203$. (a) Enlarged XANES spectrum. The data were offset for clarity. The absorption energies were listed in Table 4. 


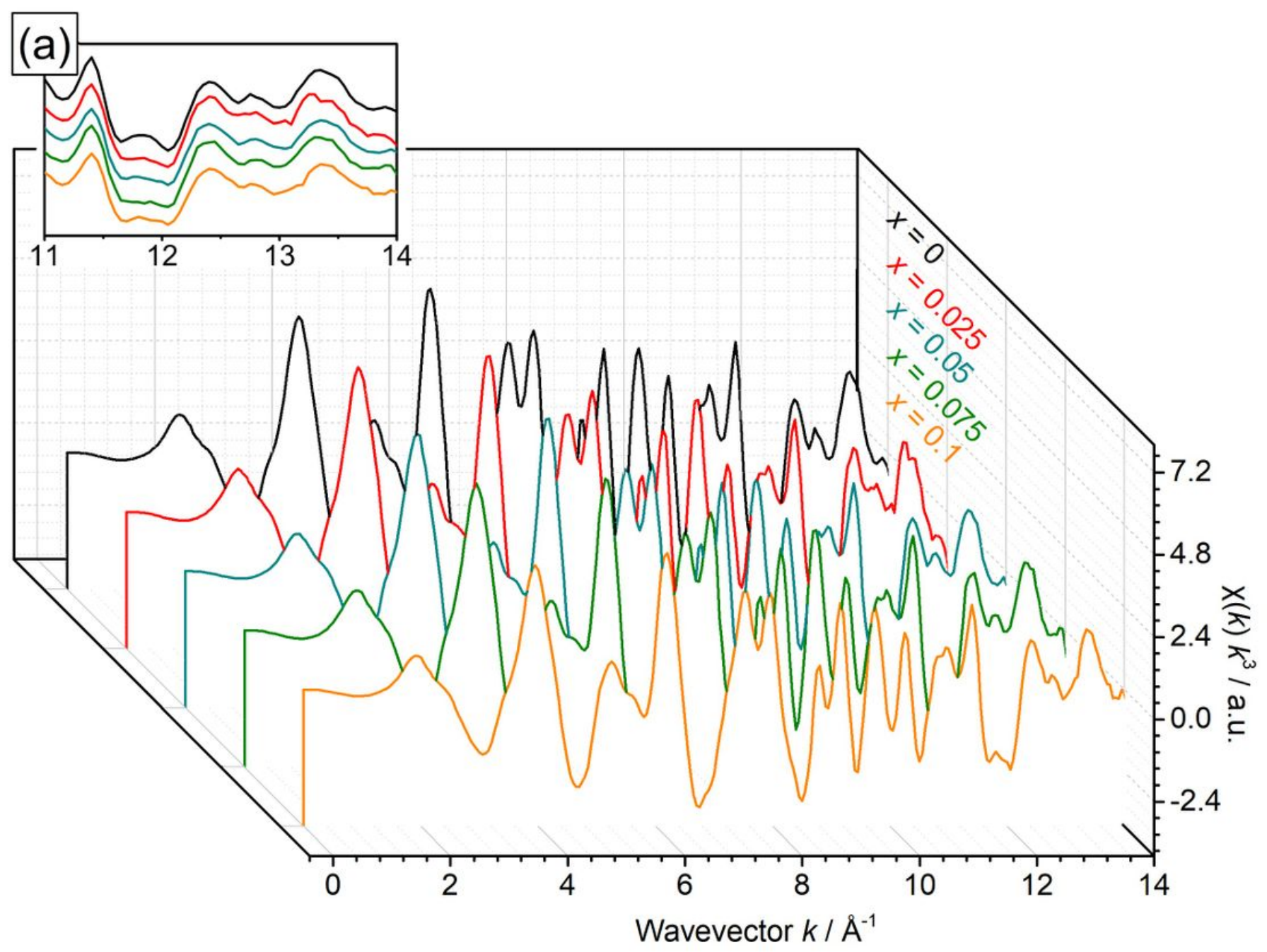

Figure 8

Y K-edge EXAFS oscillations of YFMxO with $0 \leq x \leq 0.1$. (a) Selected part of the EXAFS spectrum. The data were offset for clarity. 


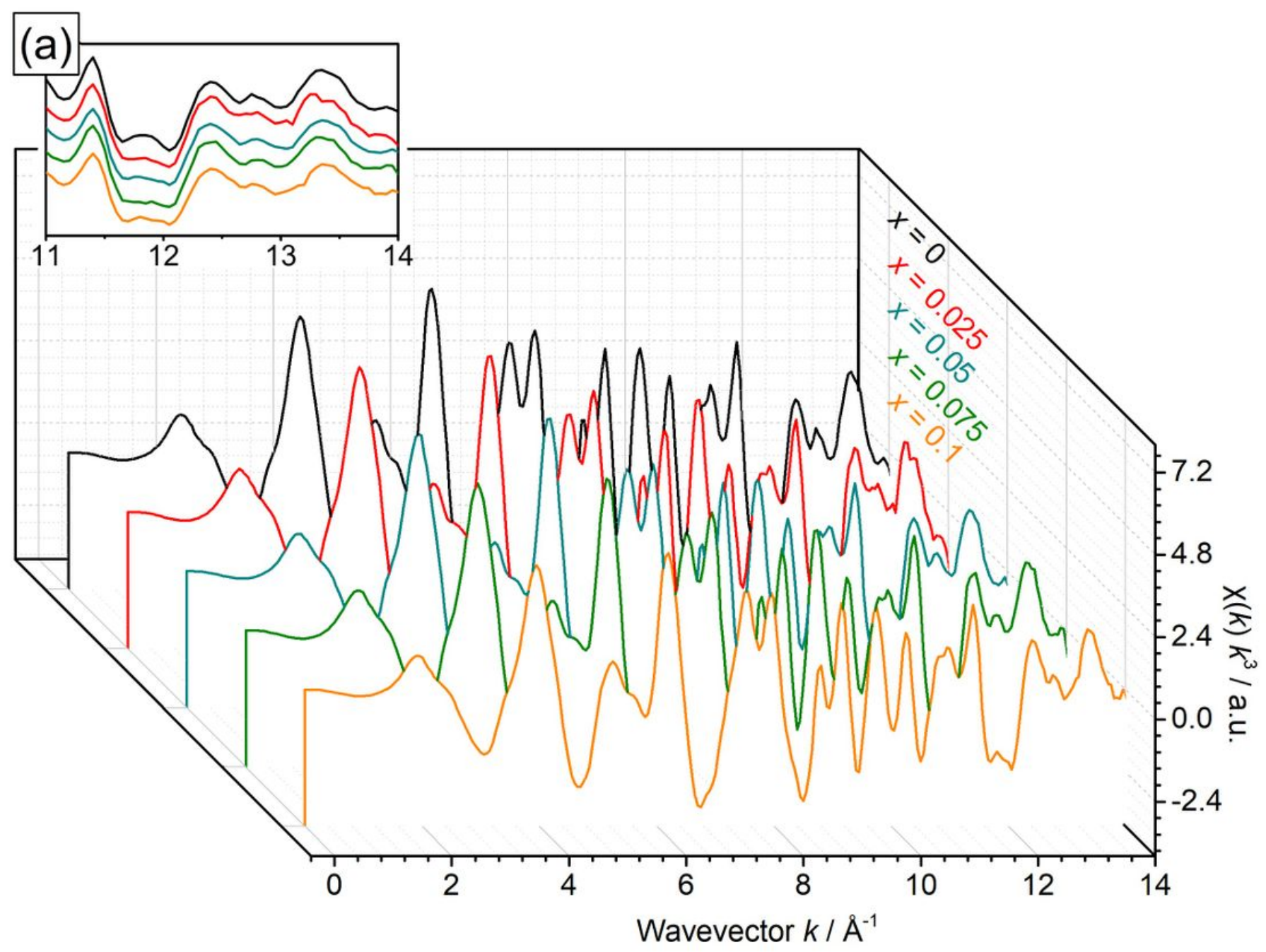

Figure 8

Y K-edge EXAFS oscillations of YFMxO with $0 \leq x \leq 0.1$. (a) Selected part of the EXAFS spectrum. The data were offset for clarity. 


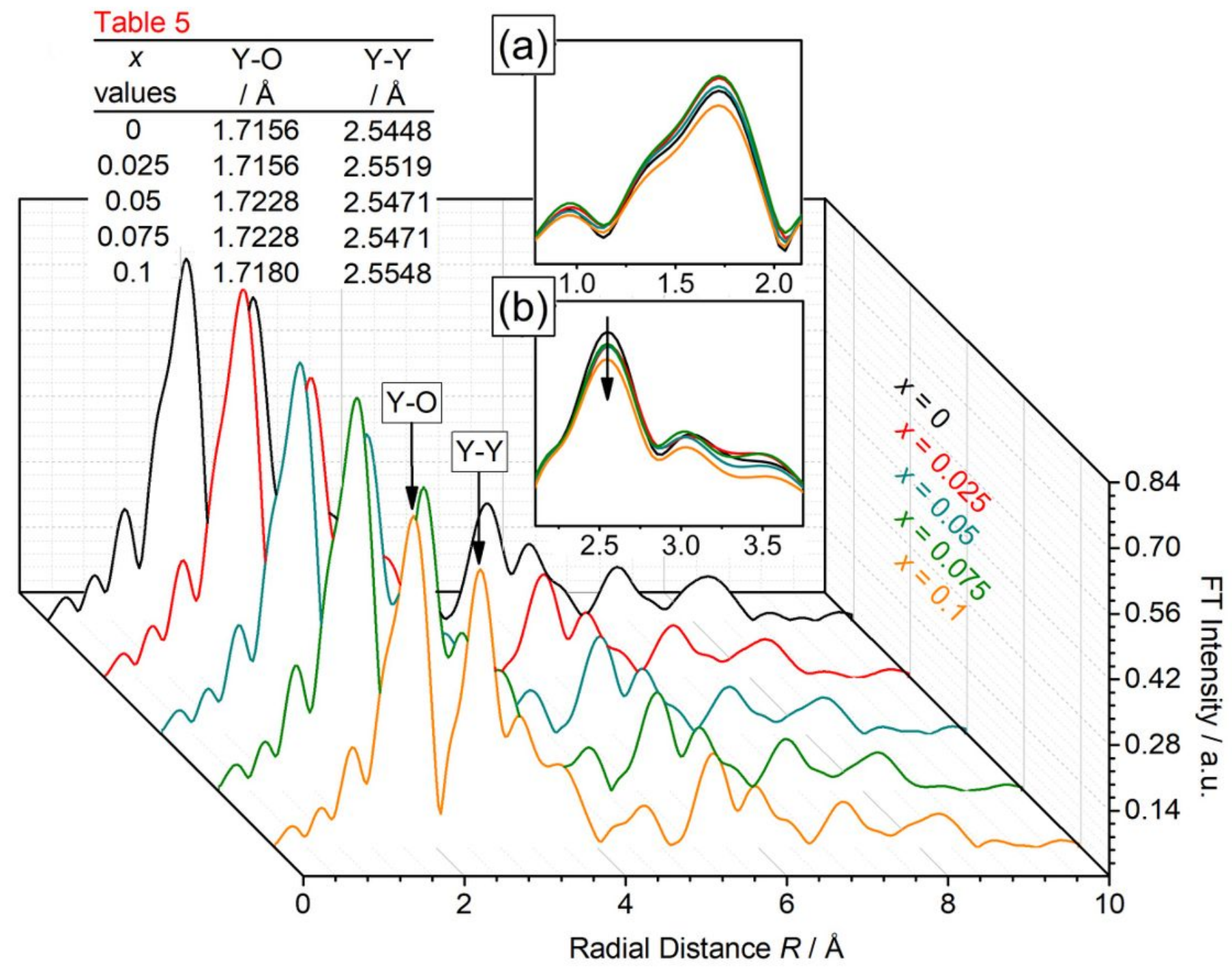

Figure 9

Fourier transform of $Y$ K-edge EXAFS functions of YFMxO with $0 \leq x \leq 0.1$. (a) Magnified EXAFS spectrum. The data were offset for clarity. The $\mathrm{Y}-\mathrm{O}$ and $\mathrm{Y}-\mathrm{Y}$ interatomic distances were listed in Table 5. 


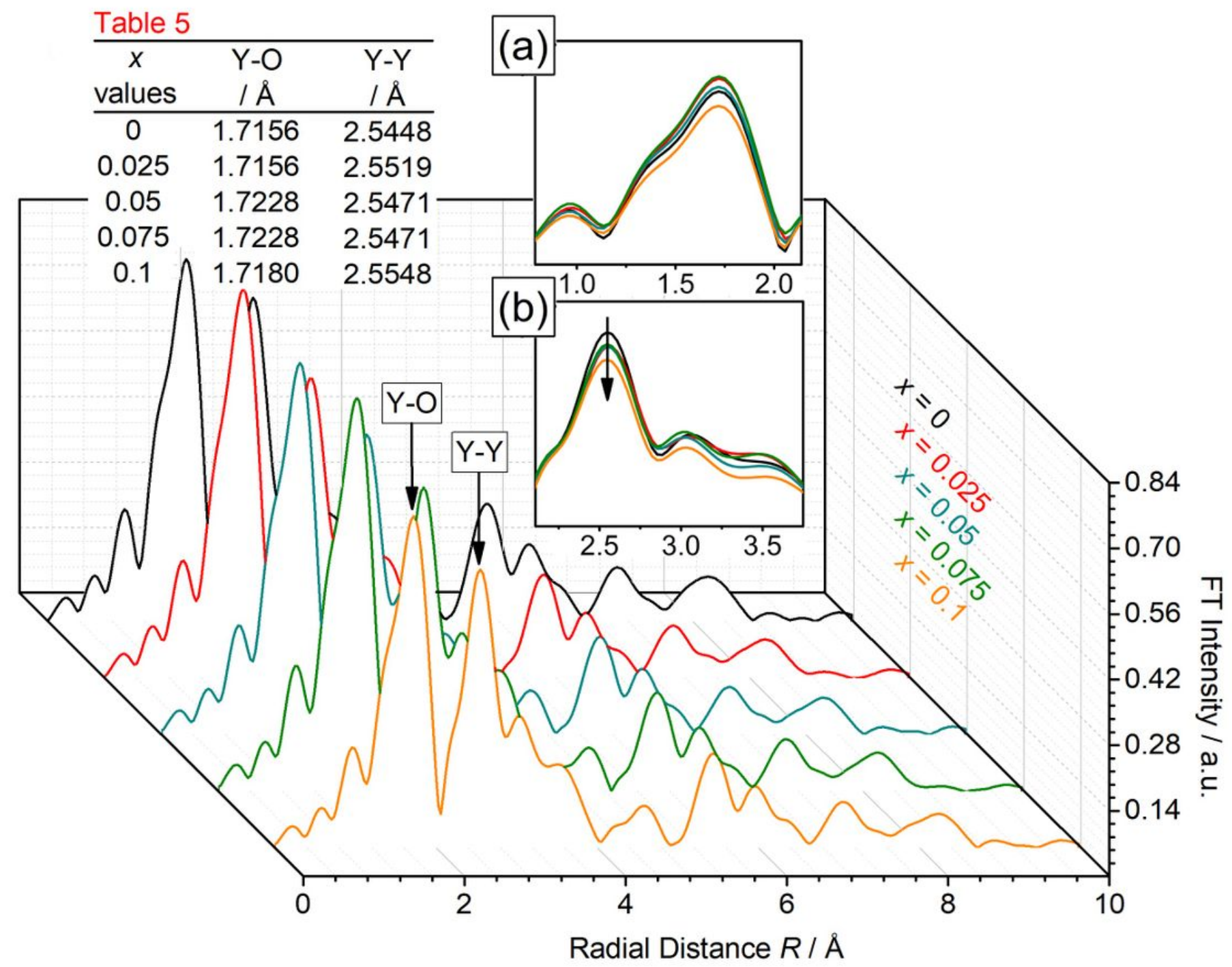

Figure 9

Fourier transform of $Y$ K-edge EXAFS functions of YFMxO with $0 \leq x \leq 0.1$. (a) Magnified EXAFS spectrum. The data were offset for clarity. The $\mathrm{Y}-\mathrm{O}$ and $\mathrm{Y}-\mathrm{Y}$ interatomic distances were listed in Table 5. 


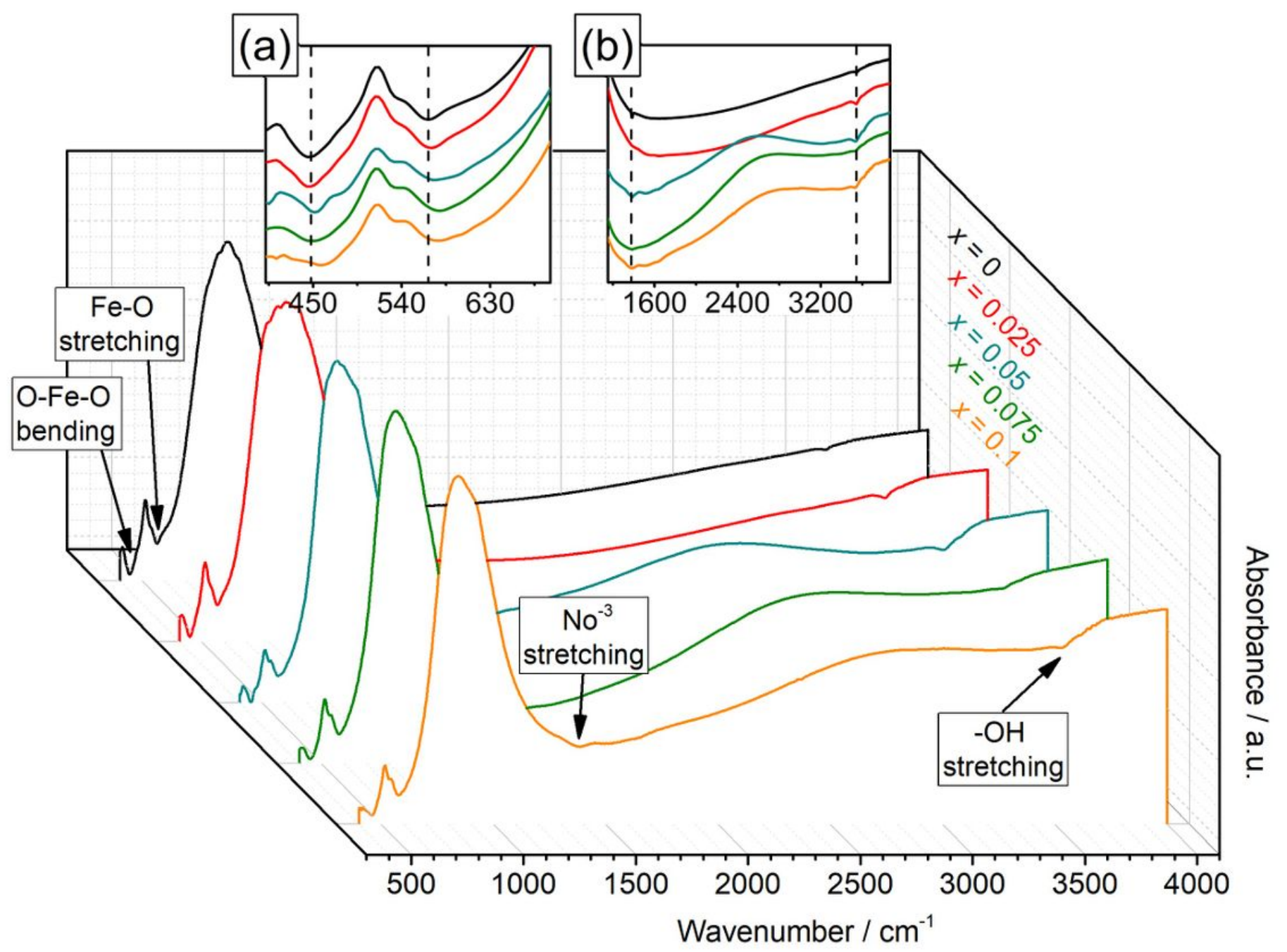

Figure 10

FT-IR spectra of YFMxO with $0 \leq x \leq 0.1$. (a)-(b) Selected part of the IR spectrum. The data were offset for clarity. 


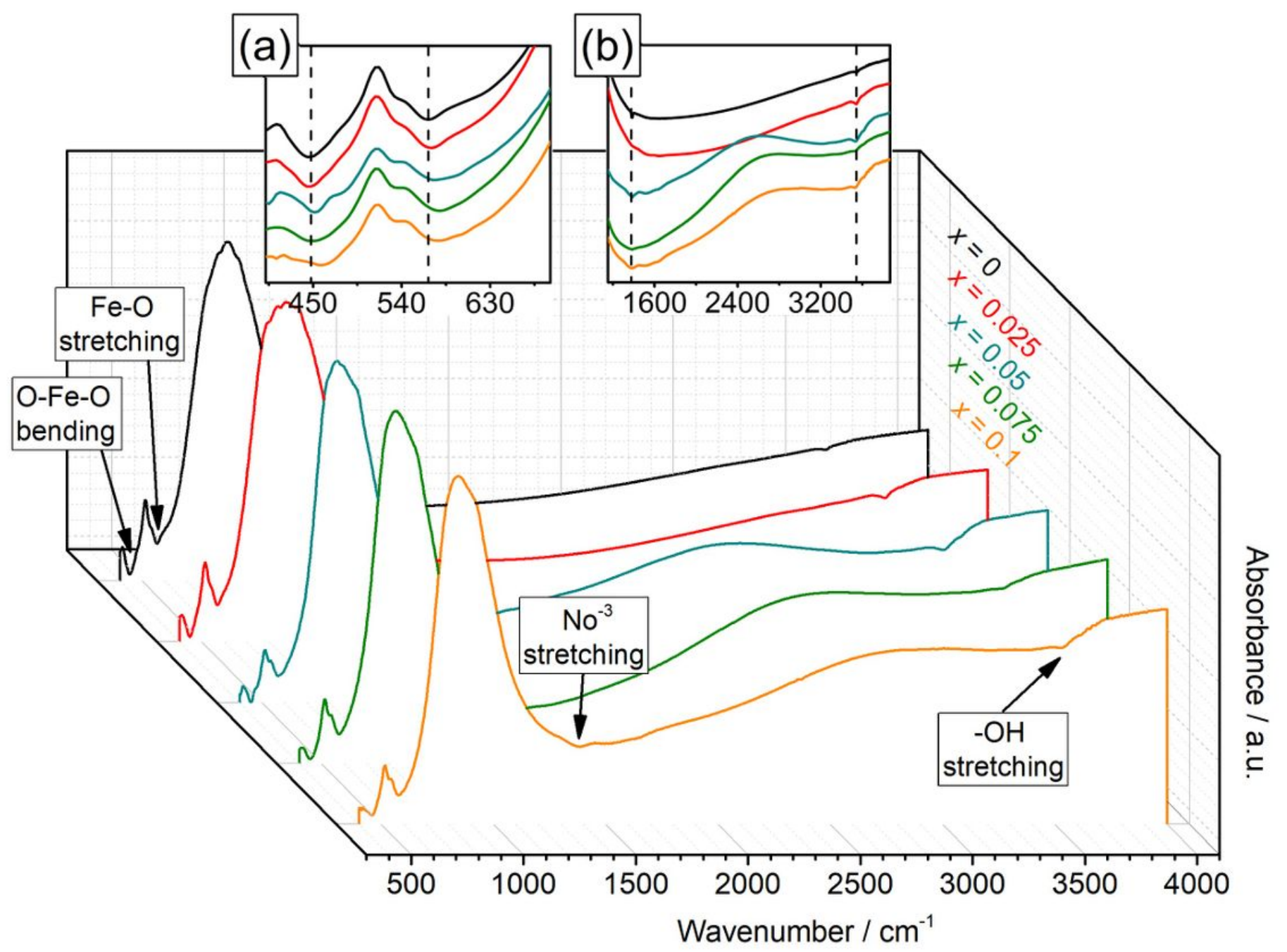

Figure 10

FT-IR spectra of YFMxO with $0 \leq x \leq 0.1$. (a)-(b) Selected part of the IR spectrum. The data were offset for clarity. 


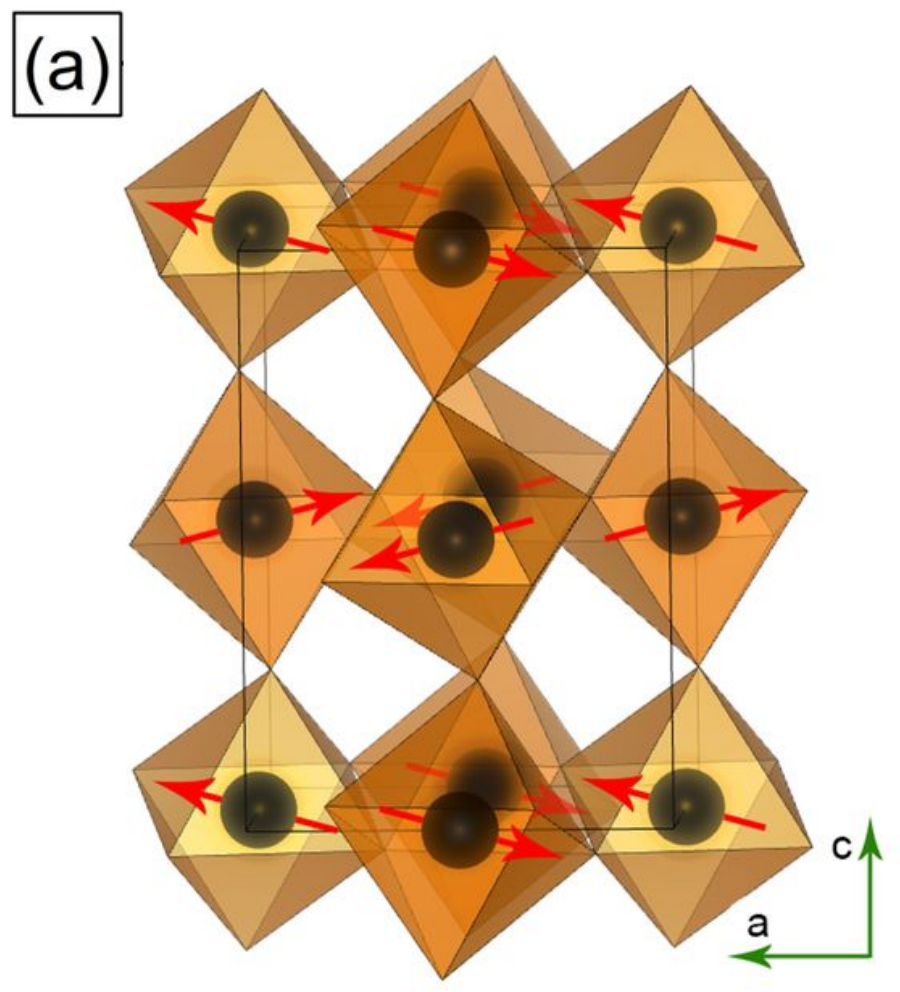

(b)

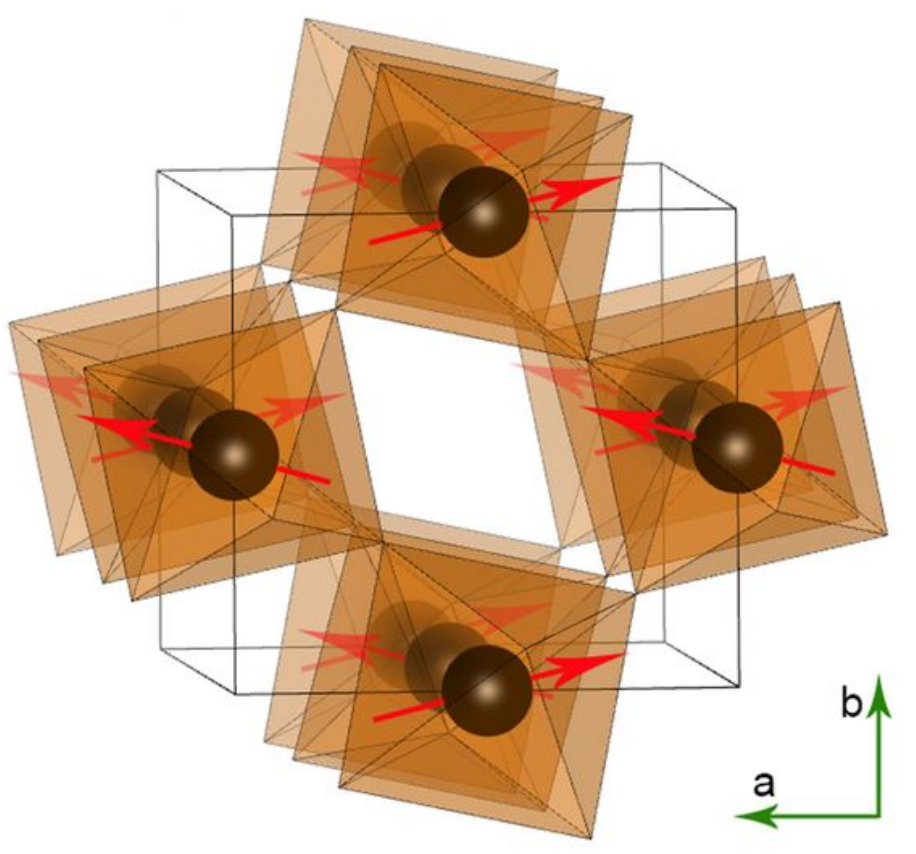

Figure 11

Magnetic unit cell of YFO from the (a) c-axis and (b) b-axis. The black spheres in the structure represents the Fe atoms.

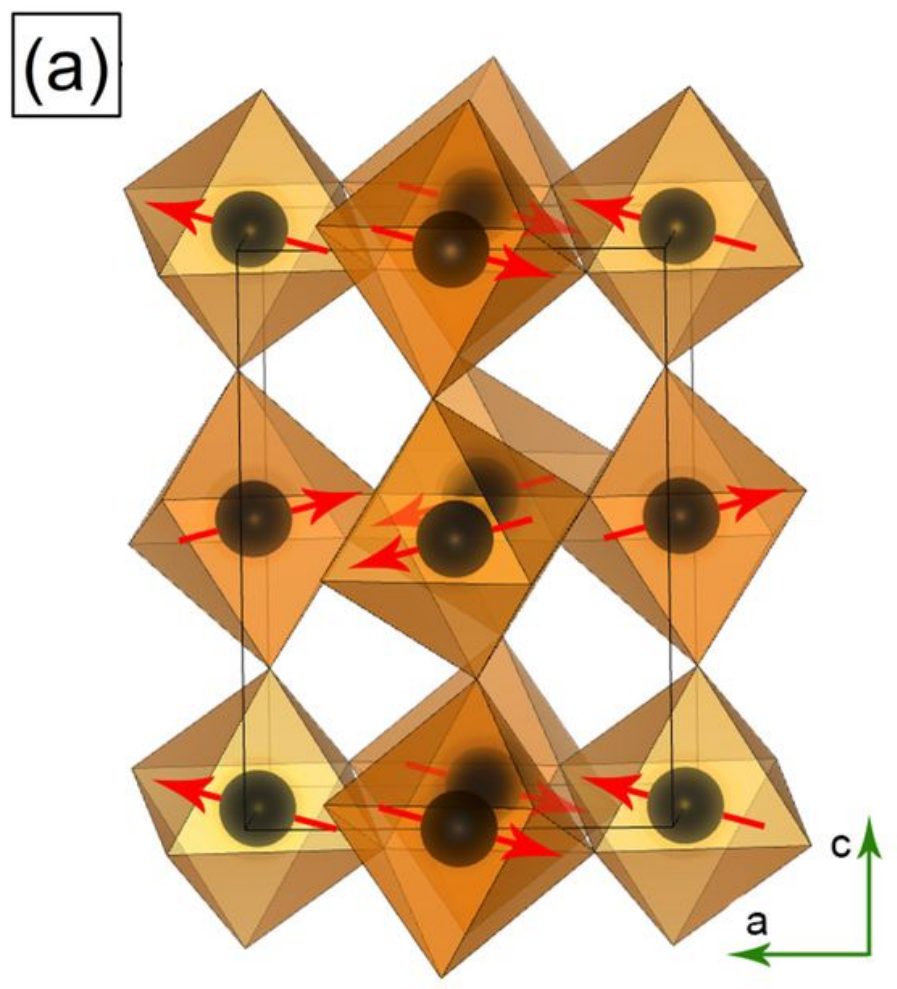

(b)

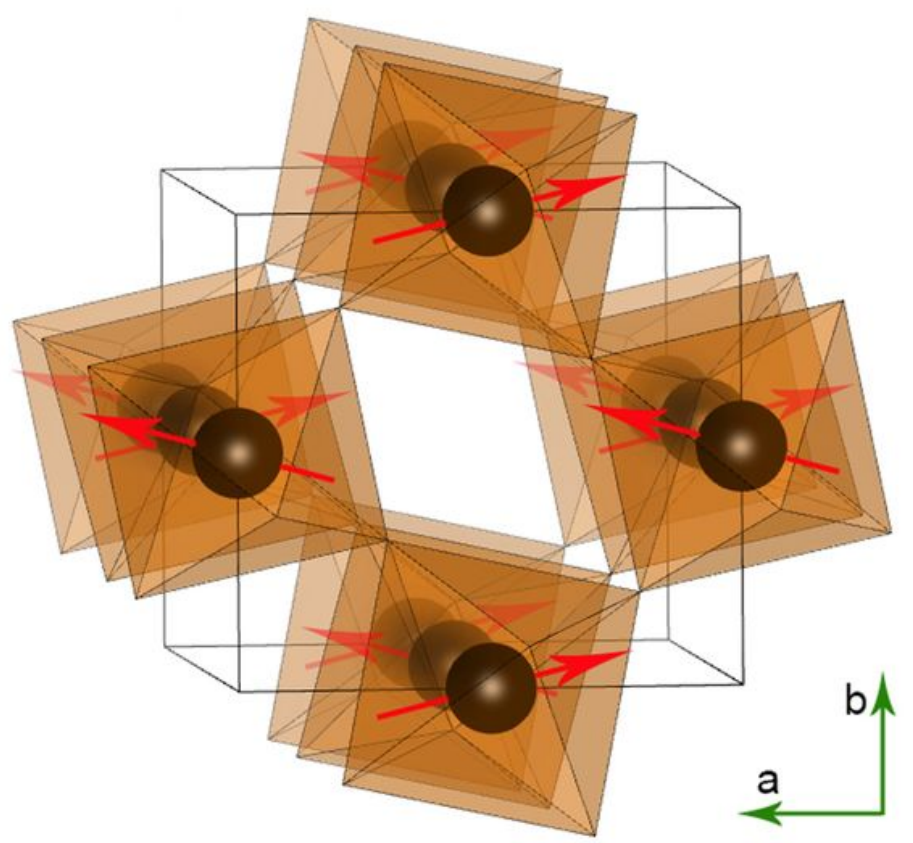


Magnetic unit cell of YFO from the (a) c-axis and (b) b-axis. The black spheres in the structure represents the Fe atoms.

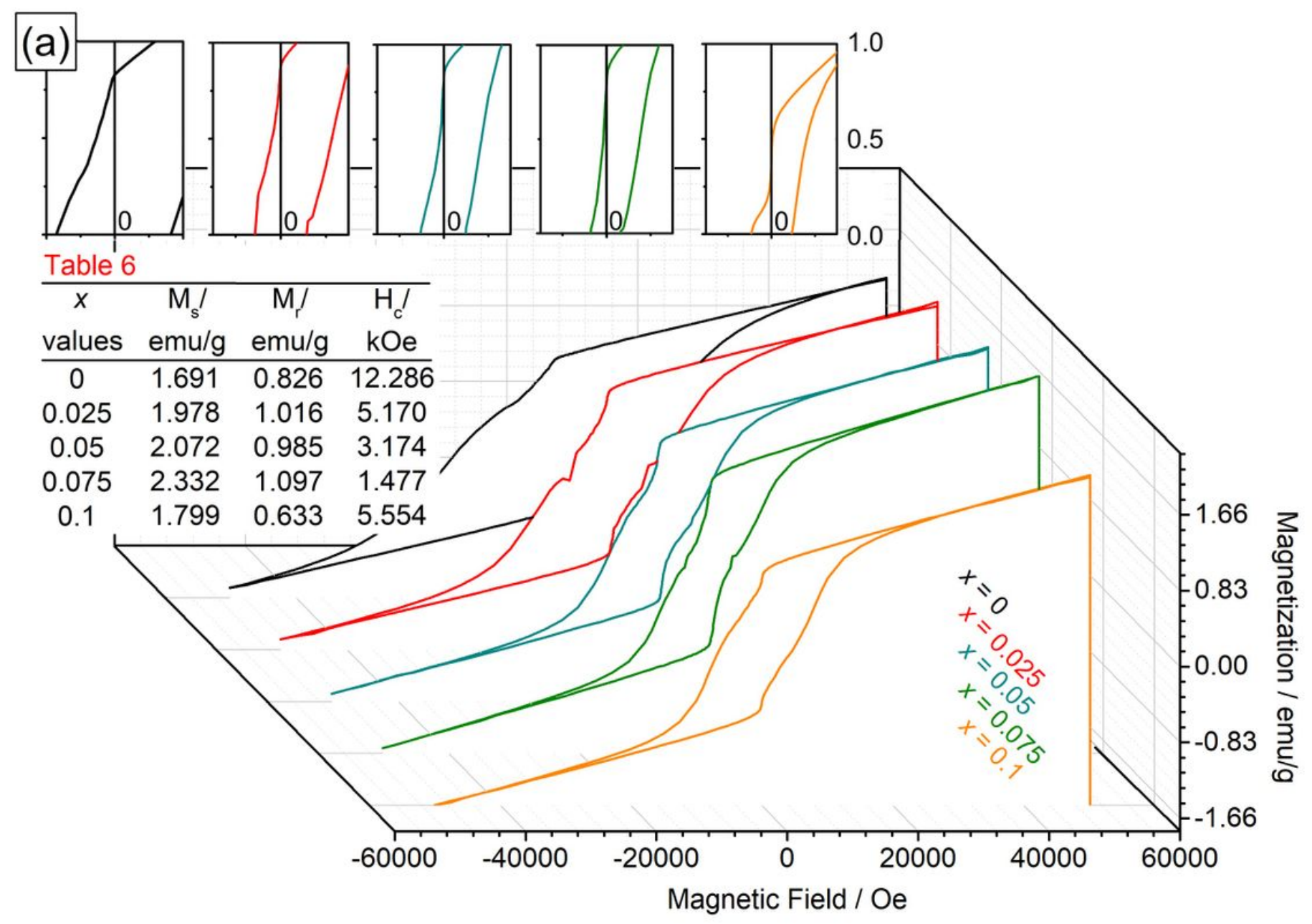

Figure 12

Magnetic hysteresis loops of YFMxO with $0 \leq x \leq 0.1$. (a) Zoom-in of M-H curves from left to right. The data were offset for clarity. The observed parameters Ms, Mr, and Hc were listed in Table 6. 


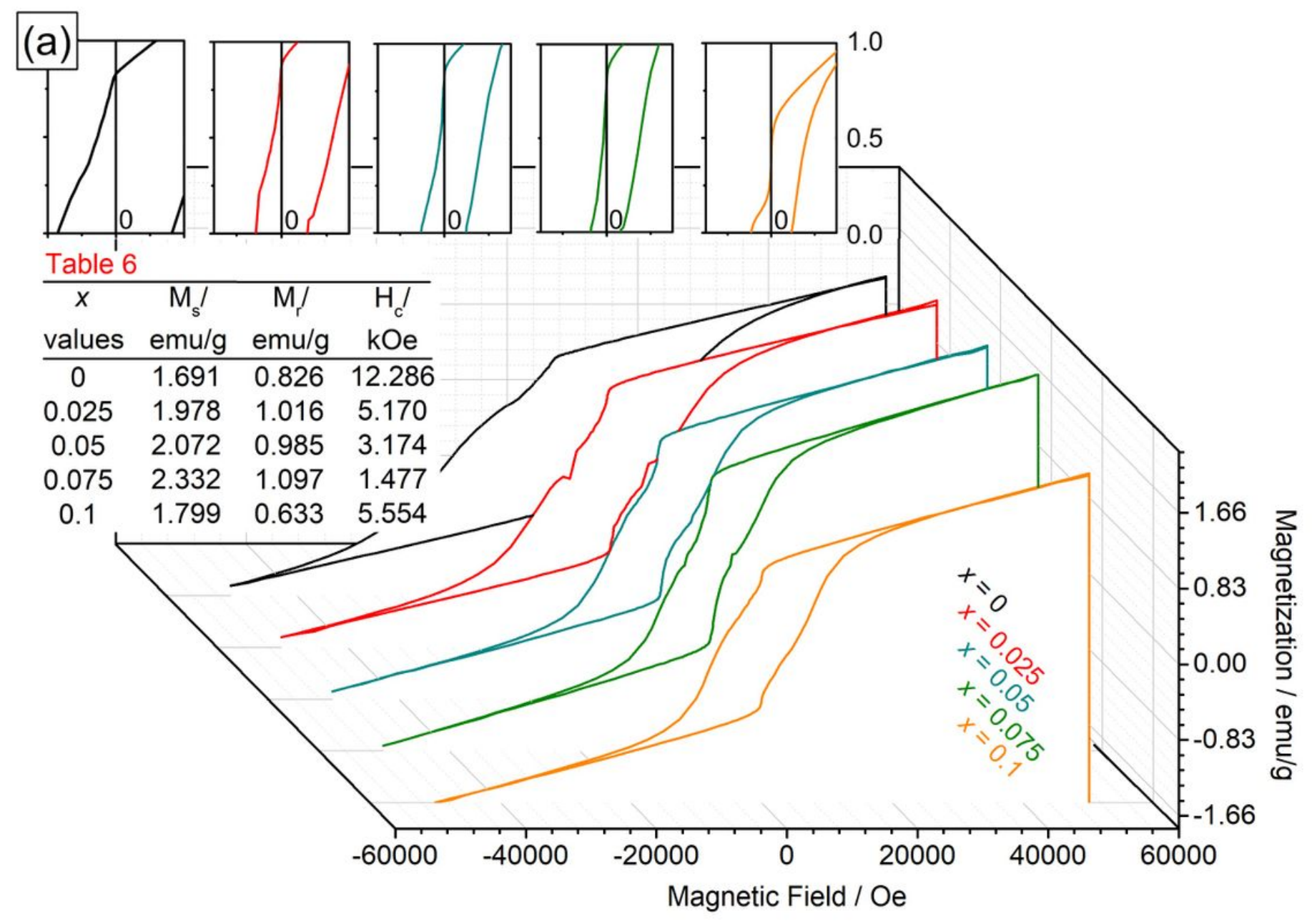

Figure 12

Magnetic hysteresis loops of YFMxO with $0 \leq x \leq 0.1$. (a) Zoom-in of M-H curves from left to right. The data were offset for clarity. The observed parameters Ms, Mr, and Hc were listed in Table 6. 